TRANSACTIONS OF THE

AMERICAN MATHEMATICAL SOCIETY

Volume 354, Number 9, Pages 3573-3600

S 0002-9947(02)03026-X

Article electronically published on May 8, 2002

\title{
ON THE PROFILE OF THE CHANGING SIGN MOUNTAIN PASS SOLUTIONS FOR AN ELLIPTIC PROBLEM
}

\author{
E. N. DANCER AND SHUSEN YAN
}

\begin{abstract}
We consider nonlinear elliptic equations with small diffusion and Dirichlet boundary conditions. We construct changing sign solutions with peaks close to the boundary and consider the location of the peak.
\end{abstract}

\section{INTRODUCTION}

Consider

$$
\begin{cases}-\varepsilon^{2} \Delta u=f(u), & \text { in } \Omega, \\ u=0, & \text { on } \partial \Omega,\end{cases}
$$

where $\Omega$ is a bounded domain in $R^{N}$ with smooth boundary, and $\varepsilon>0$ is a small number.

In recent years, there have been a lot of results on the existence and the profile of solutions for (1.1). See for example $3,5,8,8,9,11,12,13,19,21,25,26,28$. This problem arises from the biological sciences [18, 22]. It is observed that solutions of (1.1) may exhibit sharp peaks near a certain number of points. In biology, the locations of the peaks correspond to the higher concentration places of chemicals, certain populations, etc. Therefore, it is important to know the locations of the peaks of the solutions for (1.1).

In this paper, we consider a kind of nonlinearity $f(u)$, such that the mountain pass type solution for (1.1) will exhibit a new concentration phenomenon. Assume that $f(t)$ satisfies the following conditions:

$\left(f_{1}\right)$ there exists $a<b, a<0$, such that $f(a)=f(b)=0$ and $f(t)<0$ for $t \in(a, b)$;

$\left(f_{2}\right) \int_{a}^{0} f(s) d s<0$ if $b<0$

$\left(f_{3}\right) f \in C^{1}([a,+\infty)) \cap C^{2}((a,+\infty))$ and $f^{\prime \prime}(t)>0$ for all $t>a$;

$\left(f_{4}\right)$ there is $\alpha>0$, such that

$$
(t-a)^{1-\alpha} f^{\prime \prime}(t) \rightarrow c_{0}>0, \quad \text { as } t \rightarrow a+,
$$

for some $c_{0}>0$;

$\left(f_{5}\right)$ there is a $\mu>1$, such that

$$
f^{\prime}(t) t \geq \mu f(t)
$$

for $t>0$ large.

Received by the editors December 4, 2001 and, in revised form, February 19, 2002.

2000 Mathematics Subject Classification. Primary 35J60.

This work was supported by the ARC. 
$\left(f_{6}\right)|f(t)| \leq C\left(1+|t|^{p-1}\right)$ for some $p \in\left(2,2^{*}\right)$, where $2^{*}=2 N /(N-2)$ if $N \geq 3$, $2^{*}=+\infty$ if $N=1,2$.

A typical example of a function satisfying $\left(f_{1}\right)-\left(f_{6}\right)$ is

$$
f(t)=(t-a)^{p-1}-(t-a)
$$

where $2<p<2^{*}, a \in\left(-\left(\frac{p}{2}\right)^{1 /(p-2)}, 0\right)$.

Note that $\left(f_{1}\right)$ and $f^{\prime \prime}(t)>0$ for all $t>a$ imply $f^{\prime}(a)<0$. Moreover, from $f^{\prime \prime}(t)>0$, we know that $f(t)$ has exactly two zero points $a$ and $b$.

By [5], we know that $\left(f_{1}\right),\left(f_{2}\right)$ and $\left(f_{3}\right)$ guarantee the existence of a solution $\underline{u}_{\varepsilon}$, which is a local minimum of the corresponding functional of (1.1), for $\varepsilon>0$ small, with $a \leq \underline{u}_{\varepsilon} \leq 0$ and $\underline{u}_{\varepsilon} \rightarrow a$ as $\varepsilon \rightarrow 0$ on any compact subset $K$ of $\Omega$. Since $\underline{u}_{\varepsilon}$ is a local minimum, we can expect that (1.1) has a mountain pass solution $u_{\varepsilon}$.

In this paper, we shall analyse the profile of the mountain pass type solution. Let $v_{\varepsilon}=u_{\varepsilon}-\underline{u}_{\varepsilon}$. Then $v_{\varepsilon}$ satisfies

$$
\begin{cases}-\varepsilon^{2} \Delta v-f^{\prime}\left(\underline{u}_{\varepsilon}\right) v=g_{\varepsilon}(x, v), & \text { in } \Omega, \\ v=0, & \text { on } \partial \Omega,\end{cases}
$$

where

$$
g_{\varepsilon}(x, t)=f\left(\underline{u}_{\varepsilon}+t\right)-f\left(\underline{u}_{\varepsilon}\right)-f^{\prime}\left(\underline{u}_{\varepsilon}\right) t .
$$

Let

$$
\begin{gathered}
I_{\varepsilon}(v)=\frac{1}{2} \int_{\Omega}\left(\varepsilon^{2}|D v|^{2}-f^{\prime}\left(\underline{u}_{\varepsilon}\right) v^{2}\right)-\int_{\Omega} G_{\varepsilon}(x, v), \quad v \in H_{0}^{1}(\Omega), \\
G_{\varepsilon}(x, t)=F\left(\underline{u}_{\varepsilon}+t\right)-F\left(\underline{u}_{\varepsilon}\right)-f\left(\underline{u}_{\varepsilon}\right) t-\frac{1}{2} f^{\prime}\left(\underline{u}_{\varepsilon}\right) t^{2}, \quad F(t)=\int_{0}^{t} f(s) d s .
\end{gathered}
$$

We define

$$
c_{\varepsilon}=\inf _{\gamma \in \Gamma} \max _{0 \leq t \leq 1} I_{\varepsilon}(\gamma(t)),
$$

where $\Gamma=\left\{\gamma(t) \in C\left([0,1], H_{0}^{1}(\Omega)\right), \gamma(0)=0, \gamma(1)=e\right\}, e \in H_{0}^{1}(\Omega)$ is a point with $I_{\varepsilon}(e)<0$.

It follows from $\left(f_{4}\right)$ and $\left(f_{5}\right)$ that there is $\bar{\mu}_{1}>2$, such that $\bar{\mu}_{1} G_{\varepsilon}(x, t) \leq t g_{\varepsilon}(x, t)$ for $t \geq 0$ (see the proof of $(g 3)$ in section 3 ). By the mountain pass lemma of Ambrosetti and Rabinowitz [1, we know that (1.2) has a positive solution $v_{\varepsilon}$ with $I_{\varepsilon}\left(v_{\varepsilon}\right)=c_{\varepsilon}$. Thus (1.1) has a mountain pass type solution $u_{\varepsilon}=\underline{u}_{\varepsilon}+v_{\varepsilon}$. For the profile of $u_{\varepsilon}$, we have

Theorem 1.1. Let $u_{\varepsilon}=\underline{u}_{\varepsilon}+v_{\varepsilon}$ be a mountain pass solution of (1.1). There is an $\varepsilon_{0}>0$, such that for any $\varepsilon \in\left(0, \varepsilon_{0}\right]$, we have

(i) There is a $\bar{c}>0$, which is independent of $\varepsilon$, such that $\max _{x \in \bar{\Omega}} u_{\varepsilon}(x) \geq \bar{c}$.

(ii) For any local maximum point $x_{\varepsilon}$ of $u_{\varepsilon}$ with $u_{\varepsilon}\left(x_{\varepsilon}\right) \geq \tau>0$, where $\tau>0$ is some constant independent of $\varepsilon$, we have $d\left(x_{\varepsilon}, \partial \Omega\right) \leq C \varepsilon$, and for any $\theta>0$, $\left|u_{\varepsilon}(x)-\underline{u}_{\varepsilon}(x)\right| \leq C e^{-\nu\left|x-x_{\varepsilon}\right| / \varepsilon}$ if $x \in \Omega \backslash B_{\theta}\left(x_{\varepsilon}\right)$. Here $\nu>0$ is a constant.

(iii) For any sequence of $\varepsilon$, there is a subsequence $\varepsilon_{j} \rightarrow 0$, such that $x_{j} \rightarrow x_{0} \in \partial \Omega$ with $H\left(x_{0}\right)=\max _{x \in \partial \Omega} H(x)$, where $x_{j}$ is any local maximum point of $u_{\varepsilon_{j}}$ with $u_{\varepsilon_{j}}\left(x_{j}\right) \geq c^{\prime}>0, H(x)$ is the mean curvature of $\partial \Omega$ at $x$.

(iv) $\overline{\left\{x: u_{\varepsilon_{j}}(x)>0\right\}} \cap \partial \Omega \neq \emptyset$, and for any $\theta>0, \overline{\left\{x: u_{\varepsilon_{j}}(x)>0\right\}} \subset B_{\theta}\left(x_{0}\right)$, where $x_{0} \in \partial \Omega$ is the point in (iii) and $\varepsilon_{j}$ is the subsequence defined in (iii). 
By Theorem [1.1, we see that (1.1) has a changing sign mountain pass type solution which has a positive peak near a global maximum point $x_{0}$ of the mean curvature of the boundary and is negative away from a small neighbourhood of $x_{0}$.

If $f(t)$ has two zero points $b>0$ and 0 , and $f(t)<0$ for $t \in(0, b)$, Ni and Wei [26], Del Pino and Felmer [13. proved that a positive mountain pass solution of (1.1) has a unique local maximum point which locates near the center of the domain. The same result is still true if $f(t)$ has two zero points $b>a>0$, and $f(t)<0$ for $t \in(a, b)$. See [9]. Our results here look very similar to those for the Neumann problem obtained in [23, 24]. The main reason that the local maximum point of $u_{\varepsilon}$ is close to the boundary is that the corresponding problem in a half space possesses a mountain pass solution. See the result in Section 3. But unlike the Neumann problem, the local maximum point of $u_{\varepsilon}$ is inside the domain.

Our result does not claim that $u_{\varepsilon}$ has exactly one local maximum point, because, except for $N=1$, it is not clear that the solution of the form $U(x)=\underline{u}\left(x_{N}\right)+u(x)$ for problem (3.11) has exactly one local maximum point. But from the proof of the main result, it is easy to see that if $u_{\varepsilon}$ has two local maximum points $x_{\varepsilon}^{(1)}$ and $x_{\varepsilon}^{(2)}$, then $\left|x_{\varepsilon}^{(1)}-x_{\varepsilon}^{(2)}\right| \leq C \varepsilon$. Moreover, both $x_{\varepsilon}^{(1)}$ and $x_{\varepsilon}^{(2)}$ lie near (compare with $\varepsilon)$ the normal direction of $\partial \Omega$ at $\bar{x}_{\varepsilon}$, where $\bar{x}_{\varepsilon} \in \partial \Omega$ with $\left|\bar{x}_{\varepsilon}-x_{\varepsilon}^{(1)}\right|=d\left(x_{\varepsilon}^{(1)}, \partial \Omega\right)$.

If $\Omega$ is the unit ball centered at the origin, then $\underline{u}_{\varepsilon}$ is radially symmetric. Using the Mountain Pass Lemma in the radially symmetric class, we know that (1.2) has a radial solution $\bar{v}_{\varepsilon}(|x|)$. Moreover, it is easy to check that $\bar{v}_{\varepsilon}$ has a positive peak at the origin. In other words, $\bar{v}_{\varepsilon}$ has a peak at the global maximum of the distance function $d(x, \partial \Omega)$. So we may ask the question whether (1.2) always has a single peak solution with its peak near the global maximum of the distance function $d(x, \partial \Omega)$ in a general bounded domain. By Theorem 1.1, we see that this solution cannot be obtained by using the Mountain Pass Lemma in the whole space $H_{0}^{1}(\Omega)$.

We call a solution $u$ of (3.1) nondegenerate if the kernel Ker $L$ of the linear operator $L$ defined as

$$
L w=:-\Delta w-f^{\prime}\left(\underline{u}\left(x_{N}\right)\right) w-\frac{\partial g_{1}\left(x_{N}, u\right)}{\partial u} w, \quad w \in H_{0}^{1}\left(R_{+}^{N}\right),
$$

satisfies $\operatorname{Ker} L=\operatorname{span}\left\{\frac{\partial u}{\partial x_{1}}, \cdots, \frac{\partial u}{\partial x_{N-1}}\right\}$. It is an open problem whether the mountain pass solution of (3.1) is nondegenerate. If we can prove that (3.1) has a positive solution which is nondegenerate, then using the reduction method, we can easily construct various kinds of boundary peak solutions for (1.2) as in [4, 10, 17, 21]. On the other hand, we know that for certain nonlinearities $f(t)$, such as $f(t)=$ $(t-a)^{p-1}-(t-a)$, the positive solution of

$$
-\Delta u=f(u+a), \quad u \in H_{0}^{1}\left(R^{N}\right)
$$

is unique and nondegenerate. So we can prove the existence of positive interior peak solutions for (1.2) and find a lower estimate for the number of such solutions as in [7, 12, 16, 29]. Especially, (1.2) has a single peak solution with its peak near the global maximum point of $d(x, \partial \Omega)$. We shall discuss this problem briefly in this paper. Here we stress that it is the nondegeneracy not the uniqueness which is important to us. Although the results on existence of positive interior peak solutions for (1.2) look similar to those for the Neumann problem [16, 29], the locations of the peaks of the positive interior peak solutions for these two problems are different. 
It is worth pointing out that by the moving plane method of Gidas, $\mathrm{Ni}$ and Nirenberg [14, if $\Omega$ is convex, the distance of any local maximum point of a positive solution for (1.1) to the boundary of $\Omega$ has a positive lower bound which is independent of the nonlinearities and the solutions. So it is only possible for a changing sign solution to have a positive local maximum point close to the boundary of a convex domain. Therefore, the assumption $a<0$ is essential in this paper.

This paper is organized as follows. In section 2, we obtain an asymptotic expansion of the local minimum solution $\underline{u}_{\varepsilon}$ near the boundary of $\Omega$. The estimates in section 2 are essential to the proof of the main results of this paper. In section 3, we study the existence of a mountain pass solution for an elliptic problem on a half space, which corresponds to the limit problem when we blow up (1.2) at a boundary point of $\Omega$. From this mountain pass solution, we can construct an approximate solution for (1.2) and thus obtain an upper bound for $c_{\varepsilon}$. Section 4 is devoted to the proof of Theorem 1.1. In section 5, we discuss briefly the existence of interior peak solutions for (1.2). Appendix A contains a decay estimate of any positive solution of (3.1).

\section{The EXPANSION OF THE LOCAL Minimum NEAR THE BOUNDARY}

Let $\underline{u}_{\varepsilon}$ be the solution of (1.1) with $a \leq \underline{u}_{\varepsilon} \leq 0$ and $\underline{u}_{\varepsilon} \rightarrow a$ in any compact subset of $\Omega$ as $\varepsilon \rightarrow 0$. In this section, we shall obtain an asymptotic expansion for $\underline{u}_{\varepsilon}$ near the boundary of $\Omega$.

Let $\underline{u}(t)$ be the solution of

$$
\begin{cases}-u^{\prime \prime}=f(u), & t \geq 0 \\ a \leq u(t) \leq 0, & t \geq 0, \\ u(0)=0, & u(+\infty)=a .\end{cases}
$$

Then $\underline{u}(t)$ is decreasing and $|\underline{u}(t)-a| \leq C e^{-\sqrt{-f^{\prime}(a)} t}$.

Lemma 2.1. We have that there is a $c_{0}>0$, such that

$$
\int_{0}^{+\infty}\left(\left|v^{\prime}\right|^{2}-f^{\prime}(\underline{u}) v^{2}\right) \geq c_{0}\|v\|_{H_{0}^{1}\left(R_{+}^{1}\right)}^{2},
$$

for any $v \in H_{0}^{1}\left(R_{+}^{1}\right)$.

Proof. First, the proof of Proposition 2 in [6] shows that $-y^{\prime \prime}-f^{\prime}(\underline{u}) y$ with zero Dirichlet boundary condition has no nonpositive eigenvalue and this operator is seen to be Fredholm at points of the spectrum less than $-f^{\prime}(a)$. Hence we see that

$$
\inf \left\{\int_{0}^{+\infty}\left(\left|v^{\prime}\right|^{2}-f^{\prime}(\underline{u}) v^{2}\right): v \in H_{0}^{1}\left(R_{+}^{1}\right), \int_{0}^{+\infty} v^{2}=1\right\}=\lambda>0 .
$$

Thus,

$$
\int_{0}^{+\infty}\left(\left|v^{\prime}\right|^{2}-f^{\prime}(\underline{u}) v^{2}\right) \geq \lambda \int_{0}^{+\infty} v^{2}
$$


So if $c_{0}>0$ is small enough, we see

$$
\begin{aligned}
& \int_{0}^{+\infty}\left(\left|v^{\prime}\right|^{2}-f^{\prime}(\underline{u}) v^{2}\right) \\
= & c_{0} \int_{0}^{+\infty}\left|v^{\prime}\right|^{2}+\left(1-c_{0}\right) \int_{0}^{+\infty}\left|v^{\prime}\right|^{2}-\int_{0}^{+\infty} f^{\prime}(\underline{u}) v^{2} \\
\geq & c_{0} \int_{0}^{+\infty}\left|v^{\prime}\right|^{2}+\left(1-c_{0}\right)\left(\lambda \int_{0}^{+\infty} v^{2}+\int_{0}^{+\infty} f^{\prime}(\underline{u}) v^{2}\right)-\int_{0}^{+\infty} f^{\prime}(\underline{u}) v^{2} \\
= & c_{0} \int_{0}^{+\infty}\left|v^{\prime}\right|^{2}+\left(1-c_{0}\right) \lambda \int_{0}^{+\infty} v^{2}-c_{0} \int_{0}^{+\infty} f^{\prime}(\underline{u}) v^{2} \\
\geq & c_{0} \int_{0}^{+\infty}\left|v^{\prime}\right|^{2}+\left(\left(1-c_{0}\right) \lambda-C c_{0}\right) \int_{0}^{+\infty} v^{2} \\
\geq & c_{0}^{\prime}\|v\|_{H_{0}^{1}\left(R_{+}^{1}\right)}^{2} .
\end{aligned}
$$

Lemma 2.2. Let $v_{\varepsilon}$ be a solution of

$$
\begin{cases}-\varepsilon^{2} \Delta v-f^{\prime}\left(\underline{u}\left(\frac{d(x, \partial \Omega)}{\varepsilon}\right)\right) v=h, & \text { in } \Omega, \\ v=0, & \text { on } \partial \Omega .\end{cases}
$$

Then $\left|v_{\varepsilon}\right|_{\infty} \leq C|h|_{\infty}$ for some $C>0$, independent of $\varepsilon, h$ and $v_{\varepsilon}$.

Proof. We argue by contradiction. Suppose that there are $\varepsilon_{m} \rightarrow 0, h_{m}$, such that the solution $v_{m}$ of (2.3) satisfies $\left|v_{m}\right|_{\infty} \geq m\left|h_{m}\right|_{\infty}$. Without loss of generality, we assume $\left|v_{m}\right|_{\infty}=1$. Then, $\left|h_{m}\right|_{\infty} \rightarrow 0$ as $m \rightarrow+\infty$. Let $x_{m} \in \Omega$ be such that $\left|v_{m}\left(x_{m}\right)\right|=\left|v_{m}\right|_{\infty}$. Let $u_{m}(y)=v_{m}\left(\varepsilon y+x_{m}\right), \varepsilon y+x_{m} \in \Omega$.

If $\frac{d\left(x_{m}, \partial \Omega\right)}{\varepsilon_{m}} \rightarrow+\infty$, then we see that

$$
-\Delta u-f^{\prime}(a) u=0, \quad \text { in } R^{N},
$$

has a nontrivial bounded solution. By averaging over the unit sphere, we see that $-u^{\prime \prime}-\frac{N-1}{r} u^{\prime}-f^{\prime}(a) u=0$ has a bounded nontrivial solution in $R_{+}^{1}$, which is impossible since $f^{\prime}(a)<0$.

If $\frac{d\left(x_{m}, \partial \Omega\right)}{\varepsilon_{m}} \leq C$ for some $C>0$, then

$$
-\Delta u-f^{\prime}(\underline{u}) u=0, \quad \text { in } R_{+}^{N},
$$

has a nontrivial bounded solution $u$ with $\left.u\right|_{x_{N}=0}=0$. This is impossible by Proposition 2 of $[$.

Let $\psi(t)$ be the solution of

$$
\left\{\begin{array}{l}
-\psi^{\prime \prime}-f^{\prime}(\underline{u}(t)) \psi=-\underline{u}^{\prime}, \quad t>0 \\
\psi(0)=\psi(+\infty)=0
\end{array}\right.
$$

The existence of such a solution is guaranteed by Lemma [2.1. Then $\psi(t)>0$ if $t>0$ by the positivity of the operator and $\underline{u}^{\prime}<0$.

Now we are ready to obtain an asymptotic expansion for $\underline{u}_{\varepsilon}$.

Proposition 2.3. Let $\underline{u}_{\varepsilon}$ be the local minimum near a. Then

$$
\underline{u}_{\varepsilon}(x)=\underline{u}\left(\frac{d(x, \partial \Omega)}{\varepsilon}\right)+\varepsilon(N-1) \psi\left(\frac{d(x, \partial \Omega)}{\varepsilon}\right) H(\bar{x})+O\left(\varepsilon^{1+\sigma}\right),
$$


for some $\sigma>0$, where $\underline{u}$ and $\psi$ are defined by (2.1) and (2.4) respectively, and $\bar{x} \in \partial \Omega$ is a point such that $|x-\bar{x}|=d(x, \partial \Omega)$.

Proof. Define

$$
\underline{u}_{\varepsilon}^{*}= \begin{cases}\underline{u}(t), & t \in[0, \delta / \varepsilon] \\ \text { smooth, } & t \in[\delta / \varepsilon, 2 \delta / \varepsilon] \\ \underline{u}(2 \delta / \varepsilon), & t \in[2 \delta / \varepsilon,+\infty)\end{cases}
$$

In $[\delta / \varepsilon, 2 \delta / \varepsilon]$, we can choose $\underline{u}_{\varepsilon}^{*}$ such that $a \leq \underline{u}_{\varepsilon}^{*}(t) \leq 0,\left|\underline{u}_{\varepsilon}^{*}(t)-a\right| \leq C e^{-\sqrt{-f^{\prime}(a)} \delta / \varepsilon}$, $\left|D^{i} \underline{u}_{\varepsilon}^{*}(t)\right| \leq C \varepsilon^{-i} e^{-\sqrt{-f^{\prime}(a)} \delta / \varepsilon}, i=1,2$.

Let $\underline{u}_{\varepsilon}^{* *}(x)=\underline{u}_{\varepsilon}^{*}\left(\frac{d(x, \partial \Omega)}{\varepsilon}\right)$. Then $\underline{u}_{\varepsilon}^{* *}(x)$ is constant for $d(x, \partial \Omega) \geq 2 \delta$. By a simple calculation, using the equation satisfied by $\underline{u}(t)$, we see that

$$
\begin{aligned}
-\varepsilon^{2} \Delta \underline{u}_{\varepsilon}^{* *}= & -\underline{u}_{\varepsilon}^{*^{\prime \prime}}\left(\frac{d(x, \partial \Omega)}{\varepsilon}\right)-\varepsilon \underline{u}_{\varepsilon}^{*^{\prime}}\left(\frac{d(x, \partial \Omega)}{\varepsilon}\right) \Delta d(x, \partial \Omega) \\
& = \begin{cases}f\left(\underline{u}_{\varepsilon}^{*}\left(\frac{d(x, \partial \Omega)}{\varepsilon}\right)\right)-\varepsilon \underline{u}_{\varepsilon}^{*^{\prime}}\left(\frac{d(x, \partial \Omega)}{\varepsilon}\right) \Delta d(x, \partial \Omega), & \text { if } d(x, \partial \Omega) \leq \delta, \\
O\left(\varepsilon^{-2} e^{-\delta^{\prime} / \varepsilon}\right)-\varepsilon \underline{u}_{\varepsilon}^{*^{\prime}}\left(\frac{d(x, \partial \Omega)}{\varepsilon}\right) \Delta d(x, \partial \Omega), & \text { if } \delta \leq d(x, \partial \Omega) \leq 2 \delta, \\
-\varepsilon \underline{u}_{\varepsilon}^{*^{\prime}}\left(\frac{d(x, \partial \Omega)}{\varepsilon}\right) \Delta d(x, \partial \Omega), & \text { if } d(x, \partial \Omega) \geq 2 \delta, \\
= & f\left(\underline{u}_{\varepsilon}^{* *}\right)-\varepsilon \underline{u}_{\varepsilon}^{*^{\prime}}\left(\frac{d(x, \partial \Omega)}{\varepsilon}\right) \Delta d(x, \partial \Omega)+O\left(\varepsilon^{-2} e^{-\delta^{\prime \prime} / \varepsilon}\right),\end{cases}
\end{aligned}
$$

since for $d(x, \partial \Omega) \geq \delta$,

$$
f\left(\underline{u}_{\varepsilon}^{* *}\right)=f(a)+O\left(\left|\underline{u}_{\varepsilon}^{* *}-a\right|\right)=O\left(\varepsilon^{-2} e^{-\delta^{\prime \prime} / \varepsilon}\right) .
$$

Let $\xi_{\varepsilon}=\underline{u}_{\varepsilon}^{* *}-\underline{u}_{\varepsilon}$. Then

$$
\begin{aligned}
-\varepsilon^{2} \Delta \xi_{\varepsilon} & =f\left(\underline{u}_{\varepsilon}^{* *}\right)-f\left(\underline{u}_{\varepsilon}\right)-\varepsilon \underline{u}_{\varepsilon}^{*^{\prime}}\left(\frac{d(x, \partial \Omega)}{\varepsilon}\right) \Delta d(x, \partial \Omega)+O\left(\varepsilon^{-2} e^{-\delta^{\prime} / \varepsilon}\right) \\
& =-c_{\varepsilon}(x) \xi_{\varepsilon}-\varepsilon \underline{u}_{\varepsilon}^{*^{\prime}}\left(\frac{d(x, \partial \Omega)}{\varepsilon}\right) \Delta d(x, \partial \Omega)+O\left(\varepsilon^{-2} e^{-\delta^{\prime} / \varepsilon}\right),
\end{aligned}
$$

where $c_{\varepsilon}=-\int_{0}^{1} f^{\prime}\left(\tau \underline{u}_{\varepsilon}^{* *}+(1-\tau) \underline{u}_{\varepsilon}\right) d \tau$.

Let $\xi_{\varepsilon}=\varepsilon \psi_{\varepsilon}$. Then

$$
\begin{cases}-\varepsilon^{2} \Delta \psi_{\varepsilon}+c_{\varepsilon} \psi_{\varepsilon}=-\underline{u}_{\varepsilon}^{*^{\prime}}\left(\frac{d(x, \partial \Omega)}{\varepsilon}\right) \Delta d(x, \partial \Omega)+O\left(\varepsilon^{-3} e^{-\delta^{\prime} / \varepsilon}\right), & \text { in } \Omega, \\ \psi_{\varepsilon}=0, & \text { on } \partial \Omega .\end{cases}
$$

Similar to the proof of Lemma 2.2 it is not difficult to prove that $\left|\psi_{\varepsilon}\right|_{\infty} \leq C$. (Here we need to use Proposition 2.4 in [5] and Theorem 2 in [27] to prove that $c_{\varepsilon}\left(\varepsilon y+x_{\varepsilon}\right) \rightarrow-f^{\prime}\left(\underline{u}\left(x_{N}\right)\right)$ as $\varepsilon \rightarrow 0$ if $d\left(x_{\varepsilon}, \partial \Omega\right) \leq C \varepsilon$. $)$

Let

$$
\psi_{\varepsilon}^{*}(t)= \begin{cases}\psi(t), & \text { if } t \in[0, \delta / \varepsilon], \\ \text { smooth, } & \text { if } t \in[\delta / \varepsilon, 2 \delta / \varepsilon], \\ 0, & \text { if } t \in[2 \delta / \varepsilon,+\infty) .\end{cases}
$$

For any $x$ with $d(x, \partial \Omega) \leq \delta$, let $\bar{x}$ be the unique point on $\partial \Omega$ such that $|x-\bar{x}|=$ $d(x, \partial \Omega)$. Let

$$
\psi_{\varepsilon}^{* *}(x)=-(N-1) \psi_{\varepsilon}^{*}\left(\frac{d(x, \partial \Omega)}{\varepsilon}\right) H(\bar{x}),
$$


where $H(\bar{x})$ is the mean curvature of $\partial \Omega$ at $\bar{x}$. Noting that

$$
\Delta \psi_{\varepsilon}^{*}=\varepsilon^{-2} \psi_{\varepsilon}^{*^{\prime \prime}}+\varepsilon^{-1} \Delta d(x, \partial \Omega) \psi_{\varepsilon}^{*^{\prime}}=\varepsilon^{-2} \psi_{\varepsilon}^{*^{\prime \prime}}+O\left(\varepsilon^{-1}\right),
$$

we see

$$
\begin{aligned}
-\varepsilon^{2} \Delta \psi_{\varepsilon}^{* *} & =\varepsilon^{2}(N-1)\left(H(\bar{x}) \Delta \psi_{\varepsilon}^{*}+2 \varepsilon^{-1} \psi_{\varepsilon}^{*^{\prime}}\langle D d(x, \partial \Omega), D H(\bar{x})\rangle+\psi_{\varepsilon}^{*} \Delta H(\bar{x})\right) \\
& =(N-1) H(\bar{x}) \psi_{\varepsilon}^{*^{\prime \prime}}+O(\varepsilon) \\
& =(N-1)\left(-f^{\prime}\left(\underline{u}\left(\frac{d(x, \partial \Omega)}{\varepsilon}\right)\right) \psi_{\varepsilon}^{*}+\underline{u}^{\prime}\left(\frac{d(x, \partial \Omega)}{\varepsilon}\right)\right) H(\bar{x})+O(\varepsilon) \\
& =f^{\prime}\left(\underline{u}\left(\frac{d(x, \partial \Omega)}{\varepsilon}\right)\right) \psi_{\varepsilon}^{* *}+(N-1) \underline{u}^{\prime}\left(\frac{d(x, \partial \Omega)}{\varepsilon}\right) H(\bar{x})+O(\varepsilon) .
\end{aligned}
$$

Thus,

$$
\begin{aligned}
& -\varepsilon^{2} \Delta\left(\psi_{\varepsilon}^{* *}-\psi_{\varepsilon}\right)-f^{\prime}\left(\underline{u}\left(\frac{d(x, \partial \Omega)}{\varepsilon}\right)\right)\left(\psi_{\varepsilon}^{* *}-\psi_{\varepsilon}\right) \\
= & \left(f^{\prime}\left(\underline{u}\left(\frac{d(x, \partial \Omega)}{\varepsilon}\right)\right)-c_{\varepsilon}\right) \psi_{\varepsilon}+\underline{u}^{\prime}\left(\frac{d(x, \partial \Omega)}{\varepsilon}\right)((N-1) H(\bar{x})+\Delta d(x, \partial \Omega))+O(\varepsilon) \\
= & O\left(\varepsilon^{\sigma}\right),
\end{aligned}
$$

since

$$
\begin{aligned}
& f^{\prime}\left(\underline{u}\left(\frac{d(x, \partial \Omega)}{\varepsilon}\right)\right)-c_{\varepsilon} \\
= & \int_{0}^{1}\left(f^{\prime}\left(\underline{u}\left(\frac{d(x, \partial \Omega)}{\varepsilon}\right)\right)-f^{\prime}\left(\underline{u}_{\varepsilon}+\tau\left(u_{\varepsilon}^{* *}-\underline{u}_{\varepsilon}\right)\right)\right) d \tau \\
= & O\left(\left|f^{\prime}\left(\underline{u}\left(\frac{d(x, \partial \Omega)}{\varepsilon}\right)\right)-f^{\prime}\left(\underline{u}_{\varepsilon}+O(\varepsilon)\right)\right|\right)=O\left(\varepsilon^{\alpha}\right)
\end{aligned}
$$

and

$$
\begin{aligned}
& \underline{u}^{\prime}\left(\frac{d(x, \partial \Omega)}{\varepsilon}\right)(\Delta d(x, \partial \Omega)+(N-1) H(\bar{x})) \\
= & O\left(\underline{u}^{\prime}\left(\frac{d(x, \partial \Omega)}{\varepsilon}\right)|x-\bar{x}|\right)=O\left(\varepsilon^{1-\tau}\right), \quad \text { for any } \tau>0 .
\end{aligned}
$$

Here, in the last equality, we have used Lemma 14.17 in [15]. By Lemma 2.2, we obtain

$$
\left|\psi_{\varepsilon}^{* *}-\psi_{\varepsilon}\right|=O\left(\varepsilon^{\sigma}\right)
$$

for some $\sigma>0$.

\section{Existence of the MOUntain PASs SOlution in HALF SPACE}

Let $\underline{u}(t)$ be a solution of (2.1). Consider

$$
\left\{\begin{array}{l}
-\Delta u-f^{\prime}\left(\underline{u}\left(x_{N}\right)\right) u=g_{1}\left(x_{N}, u\right), \quad \text { in } R_{+}^{N}, \\
u \in H_{0}^{1}\left(R_{+}^{N}\right),
\end{array}\right.
$$

where $g_{1}\left(x_{N}, t\right)=f\left(\underline{u}\left(x_{N}\right)+t\right)-f\left(\underline{u}\left(x_{N}\right)\right)-f^{\prime}\left(\underline{u}\left(x_{N}\right)\right) t$.

Define

$$
J(u)=\frac{1}{2} \int_{R_{+}^{N}}\left(|D u|^{2}-f^{\prime}\left(\underline{u}\left(x_{N}\right)\right) u^{2}\right)-\int_{R_{+}^{N}} G_{1}\left(x_{N}, u\right),
$$


where

$$
\begin{aligned}
G_{1}\left(x_{N}, t\right) & =\int_{0}^{t} g_{1}\left(x_{N}, \tau\right) d \tau \\
& =F\left(\underline{u}\left(x_{N}\right)+t\right)-F\left(\underline{u}\left(x_{N}\right)\right)-f\left(\underline{u}\left(x_{N}\right)\right) t-\frac{1}{2} f^{\prime}\left(\underline{u}\left(x_{N}\right)\right) t^{2},
\end{aligned}
$$

and $F(t)=\int_{0}^{t} f(\tau) d \tau$.

We summarize the properties of $g_{1}\left(x_{N}, t\right)$ as follows:

(g1) $g_{1}\left(x_{N}, t\right) \geq 0$ for $t \geq 0$.

In fact, by $\left(f_{3}\right)$, we have

$$
\begin{gathered}
g_{1}\left(x_{N}, 0\right)=0, \quad \frac{\partial}{\partial t} g_{1}\left(x_{N}, 0\right)=0, \\
\frac{\partial^{2}}{\partial t^{2}} g_{1}\left(x_{N}, t\right)=f^{\prime \prime}\left(\underline{u}\left(x_{N}\right)+t\right)>0 .
\end{gathered}
$$

(g2) $\frac{g_{1}\left(x_{N}, t\right)}{t}$ is strictly increasing in $t>0$.

In fact, we have

$$
\frac{\partial}{\partial t}\left(\frac{g_{1}\left(x_{N}, t\right)}{t}\right)=\left(t \frac{\partial}{\partial t} g_{1}\left(x_{N}, t\right)-g_{1}\left(x_{N}, t\right)\right) / t^{2} .
$$

But

$$
\begin{gathered}
\left.\left(t \frac{\partial}{\partial t} g_{1}\left(x_{N}, t\right)-g_{1}\left(x_{N}, t\right)\right)\right|_{t=0}=0, \\
\frac{\partial}{\partial t}\left(t \frac{\partial}{\partial t} g_{1}\left(x_{N}, t\right)-g_{1}\left(x_{N}, t\right)\right)=t \frac{\partial^{2}}{\partial t^{2}} g_{1}\left(x_{N}, t\right)=t f^{\prime \prime}\left(\underline{u}\left(x_{N}\right)+t\right)>0,
\end{gathered}
$$

for all $t>0$. Thus $t \frac{\partial}{\partial t} g_{1}\left(x_{N}, t\right)-g_{1}\left(x_{N}, t\right)>0$ for all $t>0$.

(g3) There is a constant $\bar{\mu}>1$, such that

$$
\bar{\mu} g_{1}\left(x_{N}, t\right) \leq t \frac{\partial g_{1}\left(x_{N}, t\right)}{\partial t}, \quad \forall t \geq 0 .
$$

First, by $\left(f_{5}\right)$, we see that there is a large $T>0$ such that (3.2) holds for $t \geq T$.

Next, we claim that there is a $\theta>0$, such that

$$
t f^{\prime \prime}(\tau+t) \geq \theta\left(f^{\prime}(\tau+t)-f^{\prime}(\tau)\right), \quad \forall t \in(0, T], a \leq \tau \leq 0 .
$$

It follows from $f^{\prime \prime}(t)>0$ that (3.3) holds if $t \geq t_{0}>0$ or $\tau-a>\tau_{0}>0$, where $t_{0}$ and $\tau_{0}$ are fixed numbers. Thus it remains to prove that (3.3) holds for $t \in\left(0, t_{0}\right]$ and $\tau \in\left(a, a+\tau_{0}\right]$.

If $t \leq \tau-a$, then it follows from $\left(f_{4}\right)$ that

$$
\begin{aligned}
& t f^{\prime \prime}(\tau+t)-\theta\left(f^{\prime}(\tau+t)-f^{\prime}(\tau)\right)=t\left(f^{\prime \prime}(\tau+t)-\theta f^{\prime \prime}(\tau+\eta t)\right) \\
\geq & t\left(\frac{c_{0}^{\prime}}{(t+\tau-a)^{1-\alpha}}-\frac{\theta c_{0}^{\prime \prime}}{(\eta t+\tau-a)^{1-\alpha}}\right) \geq t\left(\frac{c_{0}^{\prime}}{(t+\tau-a)^{1-\alpha}}-\frac{\theta c_{0}^{\prime \prime}}{(\tau-a)^{1-\alpha}}\right) \\
\geq & t\left(\frac{c_{0}^{\prime}}{2^{1-\alpha}(\tau-a)^{1-\alpha}}-\frac{\theta c_{0}^{\prime \prime}}{(\tau-a)^{1-\alpha}}\right)>0
\end{aligned}
$$

if $\theta>0$ is small enough.

Suppose that $\tau-a \leq t$. From $\left(f_{4}\right)$, we can deduce

$$
\left|f^{\prime}\left(t_{1}\right)-f^{\prime}\left(t_{2}\right)\right| \leq C\left|t_{1}-t_{2}\right|^{\alpha}, \quad t_{1}, t_{2} \in[a, a+\eta] .
$$


Thus,

$$
\begin{gathered}
t f^{\prime \prime}(\tau+t)-\theta\left(f^{\prime}(\tau+t)-f^{\prime}(\tau)\right) \geq t f^{\prime \prime}(\tau+t)-C \theta t^{\alpha} \\
\geq t\left(\frac{c_{0}^{\prime}}{(t+\tau-a)^{1-\alpha}}-\frac{\theta C}{t^{1-\alpha}}\right) \geq t\left(\frac{c_{0}^{\prime}}{2^{1-\alpha} t^{1-\alpha}}-\frac{\theta C}{t^{1-\alpha}}\right)>0 .
\end{gathered}
$$

Thus we have proved $(3.3)$.

Now take $\bar{\mu}=1+\theta>1$. Let

$$
\eta\left(x_{N}, t\right)=t \frac{\partial g_{1}\left(x_{N}, t\right)}{\partial t}-\bar{\mu} g_{1}\left(x_{N}, t\right) .
$$

Then $\eta\left(x_{N}, 0\right)=0$. We have

$$
\begin{aligned}
\frac{\partial}{\partial t} \eta\left(x_{N}, t\right) & =t \frac{\partial^{2}}{\partial t^{2}} g_{1}\left(x_{N}, t\right)+(1-\bar{\mu}) \frac{\partial}{\partial t} g_{1}\left(x_{N}, t\right) \\
& =t f^{\prime \prime}\left(\underline{u}\left(x_{N}\right)+t\right)+(1-\bar{\mu})\left(f^{\prime}\left(\underline{u}\left(x_{N}\right)+t\right)-f^{\prime}\left(\underline{u}\left(x_{N}\right)\right)\right)>0,
\end{aligned}
$$

if $t \in(0, T]$. As a result, $\eta\left(x_{N}, t\right)>0$ for $t \in(0, T]$.

$(g 4)$ We have $g_{1}\left(x_{N}, t\right)=O\left(t^{2}\right)$ as $t \rightarrow 0$, and $\left|g_{1}\left(x_{N}, t\right)\right| \leq C\left(|t|^{p-1}+1\right)$.

Define

$$
c=\inf _{\gamma \in \Gamma} \max _{t \in[0,1]} J(u),
$$

where $\Gamma=\left\{\gamma(t) \in C\left([0,1], H_{0}^{1}\left(R_{+}^{N}\right)\right), \gamma(0)=0, \gamma(1)=e\right\}, e \in H_{0}^{1}\left(R_{+}^{N}\right)$ with $J(e)<0$. By $(g 2)$, it is easy to check that $c$ is independent of $e$.

It is easy to see that 3.1 is translation invariant in the $x_{i}$ direction, $i=$ $1, \cdots, N-1$. Since $\underline{u}(t) \rightarrow a$ as $t \rightarrow+\infty$, we see that the corresponding limit problem in the $x_{N}$ direction is

$$
\left\{\begin{array}{l}
-\Delta w=f(w+a), \quad \text { in } R^{N} \\
w \in H^{1}\left(R^{N}\right) .
\end{array}\right.
$$

Let

$$
A=\frac{1}{2} \int_{R^{N}}|D w|^{2}-\int_{R^{N}}\left(\int_{0}^{w} f(\tau+a) d \tau\right),
$$

where $w$ is the least energy solution of (3.5).

Using the standard concentration compactness argument 20], we can prove that (3.1) has a solution with critical value $c$ once we prove the following lemma:

Lemma 3.1. We have $c<A$.

Proof. Let $x_{l}=(0, \cdots, 0, l), w_{l}(x)=w\left(x-x_{l}\right)$. Let $P w_{l}$ be the solution of

$$
\left\{\begin{array}{l}
-\Delta v-f^{\prime}(a) v=f\left(w_{l}+a\right)-f^{\prime}(a) w_{l}, \quad \text { in } R^{N}, \\
v \in H_{0}^{1}\left(R_{+}^{N}\right) .
\end{array}\right.
$$

By the maximum principle, we have $\left|P w_{l}-w_{l}\right| \leq \max _{x_{N}=0} w_{l} \leq C e^{-\sqrt{-f^{\prime}(a)} l}$. By the definition of $c$ (see (3.4)), we have

$$
c \leq \max _{t \geq 0} J\left(t P w_{l}\right)
$$

Now we estimate $J\left(t P w_{l}\right)$.

Step 1. The estimate of $J\left(P w_{l}\right)$. 
Denote $\bar{g}(t)=f(t+a)-f^{\prime}(a) t$,

$$
\bar{G}(t)=\int_{0}^{t} \bar{g}(\tau) d \tau=F(t+a)-F(a)-\frac{1}{2} f^{\prime}(a) t^{2} .
$$

Then

$$
\begin{aligned}
J\left(P w_{l}\right)=\frac{1}{2} \int_{R_{+}^{N}}\left|D P w_{l}\right|^{2}-f^{\prime}\left(\underline{u}\left(x_{N}\right)\right)\left|P w_{l}\right|^{2}-\int_{R_{+}^{N}} G_{1}\left(x_{N}, P w_{l}\right) \\
=\frac{1}{2} \int_{R_{+}^{N}}\left(f^{\prime}(a)-f^{\prime}\left(\underline{u}\left(x_{N}\right)\right)\right)\left|P w_{l}\right|^{2}+\int_{R_{+}^{N}}\left(\frac{1}{2} \bar{g}\left(w_{l}\right) P w_{l}-G_{1}\left(x_{N}, P w_{l}\right)\right) \\
=\frac{1}{2} \int_{R_{+}^{N}}\left(f^{\prime}(a)-f^{\prime}\left(\underline{u}\left(x_{N}\right)\right)\right)\left|P w_{l}\right|^{2}+\int_{R_{+}^{N}}\left(\frac{1}{2} \bar{g}\left(w_{l}\right) P w_{l}-\bar{G}\left(P w_{l}\right)\right) \\
\quad+\int_{R_{+}^{N}}\left(\bar{G}\left(P w_{l}\right)-G_{1}\left(x_{N}, P w_{l}\right)\right) \\
=\int_{R_{+}^{N}}\left(\frac{1}{2} \bar{g}\left(w_{l}\right) P w_{l}-\bar{G}\left(P w_{l}\right)\right) \\
\quad+\int_{R_{+}^{N}}\left(F\left(a+P w_{l}\right)-F(a)-\left(F\left(\underline{u}\left(x_{N}\right)+P w_{l}\right)-F\left(\underline{u}\left(x_{N}\right)\right)-f\left(\underline{u}\left(x_{N}\right) P w_{l}\right)\right)\right. \\
=: J_{1}+J_{2},
\end{aligned}
$$

where $J_{1}$ and $J_{2}$ are defined by the last equality of the above relation.

For $J_{1}$, we have

$$
\begin{aligned}
J_{1}= & \int_{R_{+}^{N}}\left(\frac{1}{2} \bar{g}\left(w_{l}\right) w_{l}-\bar{G}\left(w_{l}\right)\right)+\frac{1}{2} \int_{R_{+}^{N}} \bar{g}\left(w_{l}\right)\left(w_{l}-P w_{l}\right) \\
& +O\left(e^{-2 \sqrt{-f^{\prime}(a) l}}\right) \\
= & \int_{R^{N}}\left(\frac{1}{2} \bar{g}\left(w_{l}\right) w_{l}-\bar{G}\left(w_{l}\right)\right)+O\left(e^{-(1+\sigma) \sqrt{-f^{\prime}(a) l}}\right) \\
& +\frac{1}{2} \int_{R_{+}^{N}}\left(-\Delta w_{l}-f^{\prime}(a) w_{l}\right)\left(w_{l}-P w_{l}\right)+O\left(e^{-2 \sqrt{-f^{\prime}(a) l}}\right) \\
= & A+\frac{1}{2} \int_{x_{N}=0}\left(\frac{\partial w_{l}}{\partial x_{N}}\left(w_{l}-P w_{l}\right)-w_{l} \frac{\partial\left(w_{l}-P w_{l}\right)}{\partial x_{N}}\right) \\
& +O\left(e^{\left.-(1+\sigma) \sqrt{-f^{\prime}(a) l}\right)}\right. \\
= & A+O\left(e^{-(1+\sigma) \sqrt{-f^{\prime}(a) l}}\right) .
\end{aligned}
$$

Now we estimate $J_{2}$. Let

$$
\begin{aligned}
K\left(t_{1}, t_{2}\right)= & F\left(a+t_{1}+t_{2}\right)-F\left(a+t_{1}\right)-f\left(a+t_{1}\right) t_{2} \\
& -\left(F\left(a+t_{2}\right)-F(a)-f(a) t_{2}\right) \\
& -\left(f\left(a+t_{2}\right) t_{1}-f(a) t_{1}-f^{\prime}(a) t_{1} t_{2}\right) .
\end{aligned}
$$

We claim that there are $C>0$ and $\sigma>0$, such that

$$
\left|K\left(t_{1}, t_{2}\right)\right| \leq C\left|t_{1}\right|^{1+\sigma}\left|t_{2}\right|^{1+\sigma} .
$$


In fact, if $\left|t_{2}\right| \leq\left|t_{1}\right|$, then

$$
\begin{aligned}
K\left(t_{1}, t_{2}\right)= & {\left[\left(f\left(a+t_{2}+\xi t_{1}\right)-f\left(a+\xi t_{1}\right)-f^{\prime}\left(a+\xi t_{1}\right) t_{2}\right)\right.} \\
& \left.-\left(f\left(a+t_{2}\right)-f(a)-f^{\prime}(a) t_{2}\right)\right] t_{1} \\
= & {\left[\left(f^{\prime}\left(a+\xi_{1} t_{2}+\xi t_{1}\right)-f^{\prime}\left(a+\xi t_{1}\right)\right)-\left(f^{\prime}\left(a+\xi_{1} t_{2}\right)-f^{\prime}(a)\right)\right] t_{1} t_{2} . }
\end{aligned}
$$

By $(f 4)$, we see $\left|f^{\prime}\left(t_{1}\right)-f^{\prime}\left(t_{2}\right)\right| \leq C\left|t_{1}-t_{2}\right|^{\alpha}$. As a result,

$$
\left|K\left(t_{1}, t_{2}\right)\right| \leq C\left|t_{2}\right|^{\alpha}\left|t_{1} t_{2}\right| \leq C\left|t_{1}\right|^{1+\alpha / 2}\left|t_{2}\right|^{1+\alpha / 2} .
$$

If $\left|t_{1}\right| \leq\left|t_{2}\right|$, then

$$
\begin{aligned}
K\left(t_{1}, t_{2}\right)= & \frac{1}{2}\left(f^{\prime}\left(a+t_{2}+\xi t_{1}\right)-f^{\prime}\left(a+\xi t_{1}\right)\right) t_{1}^{2} \\
& -\left(f^{\prime}\left(a+\xi t_{1}\right)-f^{\prime}(a)\right) t_{1} t_{2} .
\end{aligned}
$$

Thus,

$$
\begin{aligned}
K\left(t_{1}, t_{2}\right) & =C\left|t_{2}\right|^{\alpha} t_{1}^{2}+C\left|t_{1}\right|^{\alpha}\left|t_{1} t_{2}\right| \\
& \leq C\left|t_{1}\right|^{1+\sigma}\left|t_{2}\right|^{1+\sigma} .
\end{aligned}
$$

We have

$$
\begin{aligned}
J_{2}= & -\int_{R_{+}^{N}}\left(f\left(a+P w_{l}\right)-f(a)-f^{\prime}(a) P w_{l}\right)\left(\underline{u}\left(x_{N}\right)-a\right) \\
& -\int_{R_{+}^{N}} K\left(\underline{u}\left(x_{N}\right)-a, P w_{l}\right) \\
= & -\int_{R_{+}^{N}}\left(f\left(a+P w_{l}\right)-f(a)-f^{\prime}(a) P w_{l}\right)\left(\underline{u}\left(x_{N}\right)-a\right) \\
& +O\left(\int_{R_{+}^{N}}\left|\underline{u}\left(x_{N}\right)-a\right|^{1+\sigma}\left|P w_{l}\right|^{1+\sigma}\right) \\
= & -\int_{R_{+}^{N}}\left(f\left(a+P w_{l}\right)-f(a)-f^{\prime}(a) P w_{l}\right)\left(\underline{u}\left(x_{N}\right)-a\right) \\
& +O\left(\int_{R_{+}^{N}} e^{-(1+\sigma) \sqrt{-f^{\prime}(a)} x_{N}} e^{-(1+\sigma) \sqrt{-f^{\prime}(a)}\left|x-x_{l}\right|}\right) \\
= & -\int_{R_{+}^{N}}\left(f\left(a+P w_{l}\right)-f(a)-f^{\prime}(a) P w_{l}\right)\left(\underline{u}\left(x_{N}\right)-a\right)+O\left(e^{-(1+\sigma) \sqrt{-f^{\prime}(a) l}}\right) .
\end{aligned}
$$

So we have

$$
\begin{aligned}
J\left(P w_{l}\right)= & A-\int_{R_{+}^{N}}\left(f\left(a+P w_{l}\right)-f(a)-f^{\prime}(a) P w_{l}\right)\left(\underline{u}\left(x_{N}\right)-a\right) \\
& +O\left(e^{-(1+\sigma) \sqrt{-f^{\prime}(a)} l}\right) .
\end{aligned}
$$

Using the convexity of $f$, we see

$$
f(a+t)-f^{\prime}(a) t>0, \quad \forall t>0 .
$$

Noting that $\underline{u}\left(x_{N}\right)-a>0$, we obtain

$$
\begin{aligned}
& \int_{R_{+}^{N}}\left(f\left(a+P w_{l}\right)-f(a)-f^{\prime}(a) P w_{l}\right)\left(\underline{u}\left(x_{N}\right)-a\right) \\
\geq & \int_{B_{1}\left(x_{l}\right)}\left(f\left(a+P w_{l}\right)-f(a)-f^{\prime}(a) P w_{l}\right)\left(\underline{u}\left(x_{N}\right)-a\right) \\
\geq & c_{0} e^{-\sqrt{-f^{\prime}(a) l}}
\end{aligned}
$$


for some $c_{0}>0$. So we have proved $J\left(P w_{l}\right)<A$.

Step 2. The estimate of $\max _{t \geq 0} J\left(t P w_{l}\right)-J\left(P w_{l}\right)$.

Let $t_{l}$ be the maximum point of $\max _{t \geq 0} J\left(t P w_{l}\right)$. Then

$$
\left\langle J^{\prime}\left(t_{l} P w_{l}\right), P w_{l}\right\rangle=0 \text {. }
$$

We claim that $t_{l} \rightarrow 1$ as $l \rightarrow+\infty$.

In fact, from $g_{1}\left(x_{N}, t\right)=O\left(t^{2}\right)$ as $t \rightarrow 0$, and $\frac{g_{1}\left(x_{N}, t\right)}{t} \rightarrow+\infty$ as $t \rightarrow+\infty$, we see $0<t_{0} \leq t_{l} \leq T, \forall l$. Suppose that $t_{l} \rightarrow t_{\infty}$. Letting $l \rightarrow+\infty$ in (3.9), we see

$$
t_{\infty} \int_{R^{N}}|D w|^{2}=\int_{R^{N}} f\left(a+t_{\infty} w\right) w .
$$

It is easy to see that from $(g 2)$ there is exactly one $t>0$ satisfying (3.10). On the other hand, $t=1$ satisfies (3.10). Thus, $t_{\infty}=1$.

But

$$
\begin{aligned}
\left\langle J^{\prime}\left(P w_{l}\right), P w_{l}\right\rangle= & \int_{R_{+}^{N}} f^{\prime}(a) P w_{l}\left(P w_{l}-w_{l}\right)+\int_{R_{+}^{N}}\left(f\left(\underline{u}\left(x_{N}\right)\right)-f(a)\right) P w_{l} \\
& +\int_{R_{+}^{N}}\left(f\left(w_{l}+a\right)-f\left(P w_{l}+\underline{u}\left(x_{N}\right)\right)\right) P w_{l}=O\left(e^{-\sqrt{-f^{\prime}(a)} l}\right), \\
J^{\prime \prime}\left(P w_{l}\right)\left(P w_{l}, P w_{l}\right) & \\
= & \int_{R_{+}^{N}}\left(\left|D P w_{l}\right|^{2}-f^{\prime}(\underline{u})\left|P w_{l}\right|^{2}\right)-\int_{R_{+}^{N}} \frac{\partial g_{1}\left(x_{N}, P w_{l}\right)}{\partial t}\left|P w_{l}\right|^{2} \\
\leq & -c_{0}<0
\end{aligned}
$$

since $t^{2} \frac{\partial g_{1}\left(x_{N}, t\right)}{\partial t}-t g_{1}\left(x_{N}, t\right) \geq(\bar{\mu}-1) t g_{1}\left(x_{N}, t\right)$. Thus we see $t_{l}-1=O\left(e^{-\sqrt{-f^{\prime}(a)} l}\right)$. As a result,

$$
\begin{aligned}
J\left(t_{l} P w_{l}\right)-J\left(P w_{l}\right) & =\left(t_{l}-1\right)\left\langle J^{\prime}\left(P w_{l}\right), P w_{l}\right\rangle+O\left(\left|t_{l}-1\right|^{2}\right) \\
& =O\left(e^{-2 \sqrt{-f^{\prime}(a)} l}\right) .
\end{aligned}
$$

Combining Step 1 and Step 2, we obtain

$$
\begin{aligned}
\max _{t \geq 0} J\left(t P w_{l}\right) & =J\left(t_{l} P w_{l}\right)=J\left(P w_{l}\right)+O\left(e^{-2 \sqrt{-f^{\prime}(a) l}}\right) \\
& \leq A-c_{o} e^{-\sqrt{-f^{\prime}(a) l}}+O\left(e^{-(1+\sigma) \sqrt{-f^{\prime}(a) l}}\right)<A .
\end{aligned}
$$

Using Lemma 3.1 we have

Theorem 3.2. Problem (3.1) has a least energy solution $u$ with $J(u)=c$.

Proof. Let $u_{m} \in H_{0}^{1}\left(R_{+}^{N}\right)$ be a sequence with $J\left(u_{m}\right) \rightarrow c$ and $J^{\prime}\left(u_{m}\right) \rightarrow 0$ as $m \rightarrow+\infty$. By Lemma 2.1 we see that

$$
\int_{R_{+}^{N}}\left(\left|D u_{m}\right|^{2}-f^{\prime}\left(\underline{u}\left(x_{N}\right)\right) u_{m}^{2}\right) \geq c_{0}\left\|u_{m}\right\|^{2}
$$

for some $c_{0}>0$. Then by $(g 3)$, we see that $u_{m}$ is bounded in $H_{0}^{1}\left(R_{+}^{N}\right)$. Using the concentration compactness argument [20] and Lemma [3.1] we can deduce that there are $x_{m} \in\left\{x_{N}=0\right\}, m=1, \cdots$, such that $u_{m}\left(x+x_{m}\right)$ is compact in $H_{0}^{1}\left(R_{+}^{N}\right)$. As a result, (3.1) has a solution with $J(u)=c$. On the other hand, by using $(g 2)$, it is easy to check that

$$
c=\inf \left\{J(u): u \in H_{0}^{1}\left(R_{+}^{N}\right), u \neq 0,\left\langle J^{\prime}(u), u\right\rangle=0\right\} .
$$


See, for example, 24. Thus for any nontrivial solution $v$ of (3.1), $J(v) \geq c$. So $u$ is the least energy solution.

Remark 3.3. By Proposition A.1, $u(x) \leq C e^{-\sqrt{\lambda_{0}}|x|}$, for some $\lambda_{0}>0$. Since (3.1) does not depends on $x^{\prime}$, we can use the moving plane method of 14 in the directions $x_{i}, i=1, \cdots, N-1$, to prove that (3.1) has a least energy solution $u(x)=u\left(\left|x^{\prime}\right|, x_{N}\right), x^{\prime}=\left(x_{1}, \cdots, x_{N-1}\right)$.

Remark 3.4. Let $U=\underline{u}\left(x_{N}\right)+u$. Then $U$ is a solution of

$$
\begin{cases}-\Delta U=f(U), & \text { in } R_{+}^{N}, \\ U\left(x^{\prime}, 0\right)=0, & U\left(x^{\prime}, x_{N}\right) \rightarrow a, \text { uniformly in } x^{\prime} \text { as } x_{N} \rightarrow+\infty .\end{cases}
$$

Clearly, $U \geq \underline{u}$, and $U<0$ if $\left|x^{\prime}\right|$ is sufficiently large and $x_{N}>0$ is small enough. Suppose that $U$ has fixed sign. Then $\underline{u} \leq U \leq 0$. By Proposition 2.5 in [5], we have $U=\underline{u}$. This is a contradiction. So $U$ is a changing sign solution. We can also derive this result from [2] if $N=2$, or $N=3, f(0)<0$. In the next section, we shall give a direct and simple proof of this fact. See Remark 4.2

Remark 3.5. To prove that the mountain pass type solution $u_{\varepsilon}=\underline{u}_{\varepsilon}+v_{\varepsilon}$ for (1.1) has exactly one positive local maximum point, it is important to show that $U$ has exactly one positive local maximum point. It is easy to prove that this is true if $N=1$ by using the relation $\left(U^{\prime}(t)\right)^{2}-\left(U^{\prime}(0)\right)^{2}=-2 \int_{0}^{U(t)} f(\tau) d \tau$. It is an open problem whether $U$ has exactly one positive local maximum point if $N \geq 2$.

Remark 3.6. It is possible to replace the convexity assumption on $f$ by other conditions to obtain the existence result for (3.1). Under the condition that $\frac{f(t)}{t^{p} \ln ^{q} t} \rightarrow$ $a_{\infty} \in(0,+\infty)$, as $t \rightarrow+\infty$, where $1<p<\frac{N+2}{N-2}$ and $q$ is finite, or $0<A<$ $t^{-p} f(t)<B<+\infty$ for large $t$, where $1<p<\frac{N+1}{N-1}$, we can prove that the Dirichlet problem on $\left\{\left|x^{\prime}\right|<M, x_{N} \in(0, m)\right\}$ has a positive mountain pass solution $u_{M, m}\left(\left|x^{\prime}\right|, x_{N}\right)$, which are uniformly bounded (by using a blow-up argument). The idea is to let $m \rightarrow+\infty$ first, and then let $M \rightarrow+\infty$ to obtain a decaying solution for (3.1). To make this work, we need to assume $f(a+t)>f^{\prime}(a) t$ for $t>0$ and $f(t)(t-\tilde{t})>2 F(t)$ for $t>\tilde{t}$, where $F(t) \leq 0$ for $t \leq \tilde{t}$. Using the second condition, we can rewrite the energy of the solution to an integral, where the integrand is positive. Thus we can use the first condition to stop part of the solution moving to infinity in the $x_{N}$ direction. For this remark, we choose $F$ with $F(a)=0$.

\section{The LOCATion of the PEAK of the MOUNTAin PASS SOlution}

Let $\underline{u}_{\varepsilon}(x)$ be a solution of (1.1) such that $\left|\underline{u}_{\varepsilon}-a\right|$ is small on any $K \subset \subset \Omega$. Now we consider (1.2). Similar to the proof of Proposition 2 of [6], we can check that the first eigenvalue $\lambda_{\varepsilon, 1}$ of $-\varepsilon^{2} \Delta-f^{\prime}\left(\underline{u}_{\varepsilon}\right) I$ in $H_{0}^{1}(\Omega)$ satisfies $\lambda_{\varepsilon, 1} \geq \lambda_{0}>0$. Thus by a similar argument to that in the proof of Lemma 2.1, we see

$$
\int_{\Omega}\left(\varepsilon^{2}|D u|^{2}-f^{\prime}\left(\underline{u}_{\varepsilon}\right) u^{2}\right) \geq c^{\prime} \int_{\Omega}\left(\varepsilon^{2}|D u|^{2}+u^{2}\right), \quad \forall u \in H_{0}^{1}(\Omega),
$$

for some $c^{\prime}>0$. So it is easy to check that (1.2) has a positive solution $v_{\varepsilon}$ with $I_{\varepsilon}\left(v_{\varepsilon}\right)=c_{\varepsilon}$. In this section, we shall prove that all the local maximum points of $v_{\varepsilon}$ tend to the same point $x_{0}$ on the boundary, at which the mean curvature $H(x)$ attains its global maximum.

First, we have an upper bound for $c_{\varepsilon}$. 
Proposition 4.1. Let $u \in H_{0}^{1}\left(R_{+}^{N}\right)$ be any solution of (3.1) with $J(u)=c$. Then we have

$$
c_{\varepsilon} \leq \varepsilon^{N}\left(c-\varepsilon B(u) H_{M}+O\left(\varepsilon^{1+\sigma}\right)\right),
$$

where $H_{M}=\max _{x \in \partial \Omega} H(x)$, and

$$
B(u)=-\frac{1}{2} \int_{x_{N}=0} D_{x_{N}} u \underline{u}^{\prime}\left(x_{N}\right)\left|x^{\prime}\right|^{2}-\frac{1}{4} \int_{x_{N}=0}\left|D_{x_{N}} u\right|^{2}\left|x^{\prime}\right|^{2} .
$$

Moreover, we have $B(u)>0$.

Proof. Take any solution $u\left(r, x_{N}\right)$ of 2.1) with $J(u)=c$, where $r=\left|x^{\prime}\right|$. Let $x_{0} \in \partial \Omega$ be a point such that $H\left(x_{0}\right)=\max _{x \in \partial \Omega} H(x)$. After translation and rotation, we may assume that $x_{0}=0$ and

$$
\begin{gathered}
\Omega \cap B_{\delta}(0)=\left\{x: x_{N}>\varphi\left(x^{\prime}\right)\right\} \cap B_{\delta}(0), \\
\partial \Omega \cap B_{\delta}(0)=\left\{x: x_{N}=\varphi\left(x^{\prime}\right)\right\} \cap B_{\delta}(0),
\end{gathered}
$$

where $\varphi\left(x^{\prime}\right) \in C^{2}\left(R^{N-1}\right), \varphi(0)=0, D \varphi(0)=0$ and

$$
\varphi\left(x^{\prime}\right)=\frac{1}{2} \sum_{i=1}^{N-1} a_{i} x_{i}^{2}+O\left(\left|x^{\prime}\right|^{2}\right) .
$$

Let $\eta \in C_{0}^{\infty}\left(B_{\delta}(0)\right), \eta=1$ for $x \in B_{\delta / 2}(0), 0 \leq \eta \leq 1$. Define

$$
w_{\varepsilon}=\eta(x) u\left(\varepsilon^{-1} r, \varepsilon^{-1}\left(x_{N}-\varphi\left(x^{\prime}\right)\right)\right),
$$

where $r=\left|x^{\prime}\right|$. Then $w_{\varepsilon} \in H_{0}^{1}(\Omega)$. So we have

$$
c_{\varepsilon} \leq \max _{t \geq 0} I_{\varepsilon}\left(t w_{\varepsilon}\right)
$$

Now we estimate $I_{\varepsilon}\left(w_{\varepsilon}\right)$. We have

$$
\begin{aligned}
I_{\varepsilon}\left(w_{\varepsilon}\right) & =\frac{1}{2} \int_{\Omega} \varepsilon^{2}\left|D w_{\varepsilon}\right|^{2}-\int_{\Omega}\left(F\left(\underline{u}_{\varepsilon}+w_{\varepsilon}\right)-F\left(\underline{u}_{\varepsilon}\right)-f\left(\underline{u}_{\varepsilon}\right) w_{\varepsilon}\right) \\
& =I_{1}-I_{2} .
\end{aligned}
$$


Since $\underline{u}_{\varepsilon}=\underline{u}\left(\varepsilon^{-1} d(x, \partial \Omega)\right)+\varepsilon(N-1) H(\bar{x}) \psi\left(\varepsilon^{-1} d(x, \partial \Omega)\right)+O\left(\varepsilon^{1+\sigma}\right)$, where $\bar{x} \in \partial \Omega$ with $|x-\bar{x}|=d(x, \partial \Omega)$, we have

$$
\begin{aligned}
I_{2}= & \int_{\Omega}\left(F\left(\underline{u}\left(\frac{d(x, \partial \Omega)}{\varepsilon}\right)+w_{\varepsilon}\right)-F\left(\underline{u}\left(\frac{d(x, \partial \Omega)}{\varepsilon}\right)\right)-f\left(\underline{u}\left(\frac{d(x, \partial \Omega)}{\varepsilon}\right)\right) w_{\varepsilon}\right) \\
& +\varepsilon(N-1) \int_{\Omega}\left(f\left(\underline{u}\left(\frac{d(x, \partial \Omega)}{\varepsilon}\right)+w_{\varepsilon}\right)-f\left(\underline{u}\left(\frac{d(x, \partial \Omega)}{\varepsilon}\right)\right)\right. \\
& \left.-f^{\prime}\left(\underline{u}\left(\frac{d(x, \partial \Omega)}{\varepsilon}\right)\right) w_{\varepsilon}\right) \psi\left(\frac{d(x, \partial \Omega)}{\varepsilon}\right) H(\bar{x}) \\
& +O\left(\varepsilon^{N+1+\sigma}\right) \\
= & \varepsilon^{N}\left[\int_{\Omega_{\varepsilon}}\left(F\left(\underline{u}\left(d\left(x, \partial \Omega_{\varepsilon}\right)\right)+\tilde{w}_{\varepsilon}\right)-F\left(\underline{u}\left(d\left(x, \partial \Omega_{\varepsilon}\right)\right)\right)-f\left(\underline{u}\left(d\left(x, \partial \Omega_{\varepsilon}\right)\right)\right) \tilde{w}_{\varepsilon}\right)\right. \\
& +\varepsilon(N-1) \int_{\Omega_{\varepsilon}}\left(f\left(\underline{u}\left(d\left(x, \partial \Omega_{\varepsilon}\right)\right)+\tilde{w}_{\varepsilon}\right)-f\left(\underline{u}\left(d\left(x, \partial \Omega_{\varepsilon}\right)\right)\right)\right. \\
& \left.\left.-f^{\prime}\left(\underline{u}\left(d\left(x, \partial \Omega_{\varepsilon}\right)\right)\right) \tilde{w}_{\varepsilon}\right) \psi\left(d\left(x, \partial \Omega_{\varepsilon}\right)\right) H(\varepsilon \bar{x})\right] \\
& +O\left(\varepsilon^{N+1+\sigma}\right),
\end{aligned}
$$

where $\Omega_{\varepsilon}=\{x: \varepsilon x \in \Omega\}, \tilde{w}_{\varepsilon}=w_{\varepsilon}(\varepsilon x), x \in \Omega_{\varepsilon}$.

Let $\varphi_{\varepsilon}\left(x^{\prime}\right)=\varepsilon^{-1} \varphi\left(\varepsilon x^{\prime}\right)$. Let $\bar{x}_{\varepsilon} \in \partial \Omega_{\varepsilon}$ be such that $\left|x-\bar{x}_{\varepsilon}\right|=d\left(x, \partial \Omega_{\varepsilon}\right)$. Then we have $x_{i}-\bar{x}_{\varepsilon, i}+\left(x_{N}-\varphi_{\varepsilon}\left(\bar{x}^{\prime}\right)\right) D_{i} \varphi_{\varepsilon}\left(\bar{x}^{\prime}\right)=0, i=1, \cdots, N-1$. Thus $\left|x^{\prime}-\bar{x}^{\prime}\right|=O(\varepsilon)$. As a result,

$$
\begin{aligned}
d\left(x, \partial \Omega_{\varepsilon}\right) & =\left(1+\left|D \varphi_{\varepsilon}\left(\bar{x}^{\prime}\right)\right|^{2}\right)^{1 / 2}\left(x_{N}-\varphi_{\varepsilon}\left(\bar{x}^{\prime}\right)\right) \\
& =x_{N}-\varphi_{\varepsilon}\left(\bar{x}^{\prime}\right)+O\left(\left|D \varphi_{\varepsilon}\left(x^{\prime}\right)\right|^{2}\right)=x_{N}-\varphi_{\varepsilon}\left(x^{\prime}\right)+O\left(\varepsilon^{2}\right) .
\end{aligned}
$$

Thus, making the change of variable $y^{\prime}=x^{\prime}, y_{N}=x_{N}-\varphi_{\varepsilon}\left(x^{\prime}\right)$, we obtain

$$
\begin{aligned}
I_{2}= & \varepsilon^{N}\left[\int_{R_{+}^{N}}\left(F\left(\underline{u}\left(x_{N}\right)+u\right)-F\left(\underline{u}\left(x_{N}\right)\right)-f\left(\underline{u}\left(x_{N}\right)\right) u\right)\right. \\
& \left.+\varepsilon(N-1) \int_{R_{+}^{N}}\left(f\left(\underline{u}\left(x_{N}\right)+u\right)-f\left(\underline{u}\left(x_{N}\right)\right)-f^{\prime}\left(\underline{u}\left(x_{N}\right)\right) u\right) \psi\left(x_{N}\right) H(0)\right] \\
& +O\left(\varepsilon^{N+1+\sigma}\right) .
\end{aligned}
$$

Here, we have used the fact that $u$ decays exponentially. 
For the estimate of $I_{1}$, we have

$$
\begin{aligned}
& \varepsilon^{2} \int_{\Omega}\left|D w_{\varepsilon}\right|^{2}=\varepsilon^{2} \int_{\Omega \cap B_{\delta / 2}(0)}\left|D w_{\varepsilon}\right|^{2}+O\left(\varepsilon^{-\delta^{\prime} / \varepsilon}\right) \\
& =\varepsilon^{2} \int_{\Omega \cap B_{\delta / 2}(0)}\left(\left|D u\left(\frac{r}{\varepsilon}, \frac{x_{N}-\varphi\left(x^{\prime}\right)}{\varepsilon}\right)\right|^{2}\right. \\
& -2 D_{x_{N}} u\left(\frac{r}{\varepsilon}, \frac{x_{N}-\varphi\left(x^{\prime}\right)}{\varepsilon}\right) \sum_{i=1}^{N-1} D_{x_{i}} u\left(\frac{r}{\varepsilon}, \frac{x_{N}-\varphi\left(x^{\prime}\right)}{\varepsilon}\right) D_{x_{i}} \varphi\left(x^{\prime}\right) \\
& \left.+\left|D_{x_{N}} u\left(\frac{r}{\varepsilon}, \frac{x_{N}-\varphi\left(x^{\prime}\right)}{\varepsilon}\right)\right|^{2} \sum_{i=1}^{N-1}\left|D_{x_{i}} \varphi\left(x^{\prime}\right)\right|^{2}\right) \\
& +O\left(e^{-\delta^{\prime} / \varepsilon}\right) \\
& =\varepsilon^{N}\left(\int_{R_{+}^{N}}|D u|^{2}-2 \varepsilon \int_{R_{+}^{N}} D_{x_{N}} u \sum_{i=1}^{N-1} a_{i} x_{i} D_{x_{i}} u+O\left(\varepsilon^{2}\right)\right) .
\end{aligned}
$$

In the last equality, we have used again the fact that $u$ decays exponentially.

Since $u$ is a solution of $-\Delta u=f\left(\underline{u}\left(x_{N}\right)+u\right)-f\left(\underline{u}\left(x_{N}\right)\right)$, we have

$$
-\int_{R_{+}^{N}} \Delta u x_{i}^{2} D_{x_{N}} u=\int_{R_{+}^{N}}\left(f\left(\underline{u}\left(x_{N}\right)+u\right)-f\left(\underline{u}\left(x_{N}\right)\right)\right) x_{i}^{2} D_{x_{N}} u
$$

for $i=1, \cdots, N-1$.

But for each fixed $i$, we have

$$
\begin{aligned}
& -\int_{R_{+}^{N}} \Delta u x_{i}^{2} D_{x_{N}} u \\
& =-\int_{x_{N}=0} \frac{\partial u}{\partial n} x_{i}^{2} D_{x_{N}} u+2 \int_{R_{+}^{N}} D_{x_{N}} u x_{i} D_{x_{i}} u+\sum_{j=1}^{N} \int_{R_{+}^{N}} x_{i}^{2} D_{x_{j}} u D_{x_{j} x_{N}} u \\
& =\int_{x_{N}=0} D_{x_{N}} u x_{i}^{2} D_{x_{N}} u+2 \int_{R_{+}^{N}} D_{x_{N}} u x_{i} D_{x_{i}} u+\frac{1}{2} \sum_{j=1}^{N} \int_{R_{+}^{N}} x_{i}^{2} D_{x_{N}}\left|D_{x_{j}} u\right|^{2} \\
& =\frac{1}{2} \int_{x_{N}=0} x_{i}^{2}\left|D_{x_{N}} u\right|^{2}+2 \int_{R_{+}^{N}} D_{x_{N}} u x_{i} D_{x_{i}} u,
\end{aligned}
$$


since $D_{x_{j}} u=0$ if $x_{N}=0$, for $j=1, \cdots, N-1$. Here we do not use the summation convention. So

$$
\begin{aligned}
& 2 \int_{R_{+}^{N}} D_{x_{N}} u x_{i} D_{x_{i}} u \\
= & \int_{R_{+}^{N}}\left(f\left(\underline{u}\left(x_{N}\right)+u\right)-f\left(\underline{u}\left(x_{N}\right)\right)\right) x_{i}^{2} D_{x_{N}} u-\frac{1}{2} \int_{x_{N}=0} x_{i}^{2}\left|D_{x_{N}} u\right|^{2} \\
= & \int_{R_{+}^{N}} \frac{d}{d x_{N}}\left(F\left(\underline{u}\left(x_{N}\right)+u\right)-F\left(\underline{u}\left(x_{N}\right)\right)-f\left(\underline{u}\left(x_{N}\right)\right) u\right) x_{i}^{2} \\
& -\int_{R_{+}^{N}}\left(f\left(\underline{u}\left(x_{N}\right)+u\right)-f\left(\underline{u}\left(x_{N}\right)\right)-f^{\prime}\left(\underline{u}\left(x_{N}\right)\right) u\right) \underline{u}^{\prime}\left(x_{N}\right) x_{i}^{2} \\
& -\frac{1}{2} \int_{x_{N}=0} x_{i}^{2}\left|D_{x_{N}} u\right|^{2} \\
= & -\int_{R_{+}^{N}}\left(f\left(\underline{u}\left(x_{N}\right)+u\right)-f\left(\underline{u}\left(x_{N}\right)\right)-f^{\prime}\left(\underline{u}\left(x_{N}\right)\right) u\right) \underline{u}^{\prime}\left(x_{N}\right) x_{i}^{2} \\
& -\frac{1}{2} \int_{x_{N}=0} x_{i}^{2}\left|D_{x_{N}} u\right|^{2} \\
= & -\frac{1}{N-1} \int_{R_{+}^{N}}\left(f\left(\underline{u}\left(x_{N}\right)+u\right)-f\left(\underline{u}\left(x_{N}\right)\right)-f^{\prime}\left(\underline{u}\left(x_{N}\right)\right) u\right) \underline{u}^{\prime}\left(x_{N}\right)\left|x^{\prime}\right|^{2} \\
& -\frac{1}{2(N-1)} \int_{x_{N}=0}\left|x^{\prime}\right|^{2}\left|D_{x_{N}} u\right|^{2} .
\end{aligned}
$$

Combining (4.6) and (4.7), we obtain

$$
\begin{aligned}
I_{1}=\varepsilon^{N} & \frac{1}{2} \int_{R_{+}^{N}}|D u|^{2}+\frac{\varepsilon H(0)}{4} \int_{x_{N}=0}\left|x^{\prime}\right|^{2}\left|D_{x_{N}} u\right|^{2}+O\left(\varepsilon^{2}\right) \\
& \left.+\frac{\varepsilon H(0)}{2} \int_{R_{+}^{N}}\left(f\left(\underline{u}\left(x_{N}\right)+u\right)-f\left(\underline{u}\left(x_{N}\right)\right)-f^{\prime}\left(\underline{u}\left(x_{N}\right)\right) u\right) \underline{u}^{\prime}\left(x_{N}\right)\left|x^{\prime}\right|^{2}\right) .
\end{aligned}
$$

Putting (4.5) and (4.8) together, we see

$$
\begin{aligned}
I_{\varepsilon}\left(w_{\varepsilon}\right)= & \varepsilon^{N}\left(c+\frac{\varepsilon H(0)}{4} \int_{x_{N}=0}\left|x^{\prime}\right|^{2}\left|D_{x_{N}} u\right|^{2}+O\left(\varepsilon^{1+\sigma}\right)\right. \\
& +\frac{\varepsilon H(0)}{2} \int_{R_{+}^{N}}\left(f\left(\underline{u}\left(x_{N}\right)+u\right)-f\left(\underline{u}\left(x_{N}\right)\right)-f^{\prime}\left(\underline{u}\left(x_{N}\right)\right) u\right) \underline{u}^{\prime}\left(x_{N}\right)\left|x^{\prime}\right|^{2} \\
& \left.-(N-1) \varepsilon H(0) \int_{R_{+}^{N}}\left(f\left(\underline{u}\left(x_{N}\right)+u\right)-f\left(\underline{u}\left(x_{N}\right)\right)-f^{\prime}\left(\underline{u}\left(x_{N}\right)\right) u\right) \psi\left(x_{N}\right)\right) .
\end{aligned}
$$


Using the exponential decay of the solution $u, u=\psi=0$ if $x_{N}=0$, and self-adjointness of the Laplacian, we see

$$
\begin{aligned}
& \int_{R_{+}^{N}}\left(f\left(\underline{u}\left(x_{N}\right)+u\right)-f\left(\underline{u}\left(x_{N}\right)\right)-f^{\prime}\left(\underline{u}\left(x_{N}\right)\right) u\right) \psi\left(x_{N}\right) \\
= & \int_{R_{+}^{N}}\left(-\Delta \psi-f^{\prime}\left(\underline{u}\left(x_{N}\right)\right) \psi\right) u \\
= & -\int_{R_{+}^{N}} \underline{u}^{\prime}\left(x_{N}\right) u .
\end{aligned}
$$

Similarly, we have

$$
\begin{aligned}
& \int_{R_{+}^{N}}\left(f\left(\underline{u}\left(x_{N}\right)+u\right)-f\left(\underline{u}\left(x_{N}\right)\right)\right) \underline{u}^{\prime}\left(x_{N}\right)\left|x^{\prime}\right|^{2} \\
= & -\int_{R_{+}^{N}} \Delta u \underline{u}^{\prime}\left(x_{N}\right)\left|x^{\prime}\right|^{2} \\
= & -\int_{x_{N}=0} \frac{\partial u}{\partial n} \underline{u}^{\prime}\left(x_{N}\right)\left|x^{\prime}\right|^{2}-\int_{R_{+}^{N}} u \Delta\left(\underline{u}^{\prime}\left(x_{N}\right)\left|x^{\prime}\right|^{2}\right) \\
= & \int_{x_{N}=0} D_{x_{N}} u \underline{u}^{\prime}\left(x_{N}\right)\left|x^{\prime}\right|^{2}-\int_{R_{+}^{N}} u\left(\underline{u}^{\prime \prime \prime}\left|x^{\prime}\right|^{2}+2(N-1) \underline{u}^{\prime}\left(x_{N}\right)\right) \\
= & \int_{x_{N}=0} D_{x_{N}} u \underline{u}^{\prime}\left(x_{N}\right)\left|x^{\prime}\right|^{2}+\int_{R_{+}^{N}} u\left(f^{\prime}\left(\underline{u}\left(x_{N}\right)\right) u^{\prime}\left(x_{N}\right)\left|x^{\prime}\right|^{2}-2(N-1) \underline{u}^{\prime}\left(x_{N}\right)\right) .
\end{aligned}
$$

As a result,

$$
\begin{aligned}
& \int_{R_{+}^{N}}\left(f\left(\underline{u}\left(x_{N}\right)+u\right)-f\left(\underline{u}\left(x_{N}\right)\right)-f^{\prime}\left(\underline{u}\left(x_{N}\right)\right) u\right) \underline{u}^{\prime}\left(x_{N}\right)\left|x^{\prime}\right|^{2} \\
= & \int_{x_{N}=0} D_{x_{N}} u \underline{u}^{\prime}\left(x_{N}\right)\left|x^{\prime}\right|^{2}-2(N-1) \int_{R_{+}^{N}} u \underline{u}^{\prime}\left(x_{N}\right) .
\end{aligned}
$$

Combining (4.9), (4.10) and (4.11), we obtain

$$
I_{\varepsilon}\left(w_{\varepsilon}\right)=\varepsilon^{N}\left(c-\varepsilon B(u) H(0)+O\left(\varepsilon^{1+\sigma}\right)\right),
$$

where

$$
B(u)=-\frac{1}{2} \int_{x_{N}=0} D_{x_{N}} u \underline{u}^{\prime}(0)\left|x^{\prime}\right|^{2}-\frac{1}{4} \int_{x_{N}=0}\left|D_{x_{N}} u\right|^{2}\left|x^{\prime}\right|^{2} .
$$

It is easy to check that $\left\langle I_{\varepsilon}^{\prime}\left(w_{\varepsilon}\right), w_{\varepsilon}\right\rangle=O\left(\varepsilon^{N+1}\right), I_{\varepsilon}^{\prime \prime}\left(w_{\varepsilon}\right)\left(w_{\varepsilon}, w_{\varepsilon}\right) \leq-c_{0} \varepsilon^{N}<$ 0 . Thus, similar to the proof of step 2 of Lemma 3.1, we see that if $t_{\varepsilon}$ achieves $\max _{t \geq 0} I_{\varepsilon}\left(t w_{\varepsilon}\right)$, then $t_{\varepsilon}=1+O(\varepsilon)$. So

$$
\begin{aligned}
I_{\varepsilon}\left(t_{\varepsilon} w_{\varepsilon}\right) & =I_{\varepsilon}\left(w_{\varepsilon}\right)+\left(t_{\varepsilon}-1\right)\left\langle I_{\varepsilon}^{\prime}\left(w_{\varepsilon}\right), w_{\varepsilon}\right\rangle+O\left(\varepsilon^{N}\left|t_{\varepsilon}-1\right|^{2}\right) \\
& =I_{\varepsilon}\left(w_{\varepsilon}\right)+O\left(\varepsilon^{N+2}\right) .
\end{aligned}
$$

Thus, the upper bound follows from (4.12) and (4.13).

It remains to prove that $B(u)>0$. Let $U=\underline{u}+u$. Then $U$ satisfies $-\Delta U=f(U)$ on $R_{+}^{N}, U=0$ on $x_{N}=0$ and $U \rightarrow a<0$ as $x_{N} \rightarrow+\infty$. It is easy to check that

$$
B(u)=\frac{1}{4} \int_{x_{N}=0}\left(\underline{u}^{\prime}(0)^{2}-\left|D_{x_{N}} U\left(x^{\prime}, 0\right)\right|^{2}\right)\left|x^{\prime}\right|^{2} .
$$


First we claim that

$$
\int_{x_{N}=0}\left(\underline{u}^{\prime}(0)^{2}-\left|D_{x_{N}} U\left(x^{\prime}, 0\right)\right|^{2}\right)=0 .
$$

Multiplying $-\Delta U=f(U)$ by $D_{x_{N}} U$ and integrating over $B_{R}^{N-1}(0) \times[0,+\infty)$, where $B_{R}^{N-1}(0)$ is the ball in $R^{N-1}$, centered at the origin with radius $R$, we obtain

$$
\begin{aligned}
& -\sum_{i=1}^{N-1} \int_{B_{R}^{N-1}(0) \times[0,+\infty)} U_{x_{i} x_{i}} U_{x_{N}}+\frac{1}{2} \int_{B_{R}^{N-1}(0)} U_{x_{N}}^{2}\left(x^{\prime}, 0\right) \\
= & \int_{B_{R}^{N-1}(0)} \int_{0}^{+\infty} \frac{d}{d x_{N}} F(U) d x_{N} d x^{\prime}=\int_{B_{R}^{N-1}(0)} F(a)=\int_{B_{R}^{N-1}(0)} \int_{0}^{a} f(\tau) d \tau .
\end{aligned}
$$

But $\frac{1}{2}\left|\underline{u}^{\prime}(0)\right|^{2}=\int_{0}^{a} f(\tau) d \tau$. So we have

$$
\int_{B_{R}^{N-1}(0)}\left(\underline{u}^{\prime}(0)^{2}-\left|D_{x_{N}} U\left(x^{\prime}, 0\right)\right|^{2}\right)=-2 \sum_{i=1}^{N-1} \int_{B_{R}^{N-1}(0) \times[0,+\infty)} U_{x_{i} x_{i}} U_{x_{N}} .
$$

On the other hand, we have

$$
\begin{aligned}
& \int_{B_{R}^{N-1}(0)} U_{x_{i} x_{i}} U_{x_{N}} d x^{\prime}=\int_{\partial B_{R}^{N-1}(0)} n_{i} U_{x_{i}} U_{x_{N}}-\int_{B_{R}^{N-1}(0)} U_{x_{i}} U_{x_{N} x_{i}} d x^{\prime} \\
= & \int_{\partial B_{R}^{N-1}(0)} n_{i} U_{x_{i}} U_{x_{N}}-\frac{1}{2} \frac{d}{d x_{N}} \int_{B_{R}^{N-1}(0)} U_{x_{i}}^{2} d x^{\prime} .
\end{aligned}
$$

So

$$
\int_{B_{R}^{N-1}(0) \times[0,+\infty)} U_{x_{i} x_{i}} U_{x_{N}}=\int_{\partial B_{R}^{N-1}(0) \times[0,+\infty)} n_{i} U_{x_{i}} U_{x_{N}} \rightarrow 0
$$

as $R \rightarrow+\infty$, since $U_{x_{i}}=u_{x_{i}}$ decays exponentially as $|x| \rightarrow+\infty$. Thus (4.14) follows.

Now we prove that $B(u)>0$. Since $U\left(\left|x^{\prime}\right|, x_{N}\right)$ is a decreasing function of $\left|x^{\prime}\right|$, we see that $U_{x_{N}}\left(\left|x^{\prime}\right|, 0\right)$ is nonincreasing in $\left|x^{\prime}\right|$. Let $r_{0}$ be such that $\underline{u}^{\prime}(0)^{2}-$ $\left|D_{x_{N}} U\left(x^{\prime}, 0\right)\right|^{2} \geq 0$ if $\left|x^{\prime}\right| \geq r_{0}, \underline{u}^{\prime}(0)^{2}-\left|D_{x_{N}} U\left(x^{\prime}, 0\right)\right|^{2} \leq 0$ if $\left|x^{\prime}\right| \leq r_{0}$. Since $u_{x_{N}}\left(x^{\prime}, 0\right)$ tends to zero as $\left|x^{\prime}\right| \rightarrow+\infty$ and $u_{x_{N}}\left(x^{\prime}, 0\right) \geq 0$, we see that $\underline{u}^{\prime}(0)^{2}-$ $\left|D_{x_{N}} U\left(x^{\prime}, 0\right)\right|^{2}>0$ if $\left|x^{\prime}\right|$ is large. So $r_{0}<+\infty$. Hence, by (4.14), we obtain

$$
\begin{aligned}
B(u)= & \int_{\left|x^{\prime}\right| \leq r_{0}}\left(\underline{u}^{\prime}(0)^{2}-\left|D_{x_{N}} U\left(x^{\prime}, 0\right)\right|^{2}\right)\left|x^{\prime}\right|^{2} \\
& +\int_{\left|x^{\prime}\right| \geq r_{0}}\left(\underline{u}^{\prime}(0)^{2}-\left|D_{x_{N}} U\left(x^{\prime}, 0\right)\right|^{2}\right)\left|x^{\prime}\right|^{2} \\
\geq & \int_{\left|x^{\prime}\right| \leq r_{0}}\left(\underline{u}^{\prime}(0)^{2}-\left|D_{x_{N}} U\left(x^{\prime}, 0\right)\right|^{2}\right) r_{0}^{2} \\
& +\int_{\left|x^{\prime}\right| \geq r_{0}}\left(\underline{u}^{\prime}(0)^{2}-\left|D_{x_{N}} U\left(x^{\prime}, 0\right)\right|^{2}\right)\left|x^{\prime}\right|^{2} \\
= & \int_{\left|x^{\prime}\right| \geq r_{0}}\left(\underline{u}^{\prime}(0)^{2}-\left|D_{x_{N}} U\left(x^{\prime}, 0\right)\right|^{2}\right)\left(\left|x^{\prime}\right|^{2}-r_{0}^{2}\right)>0 .
\end{aligned}
$$

Remark 4.2. Since $U_{x_{N}}\left(x^{\prime}, 0\right) \geq \underline{u}^{\prime}(0)$, it is easy to see from (4.14) that

$$
U_{x_{N}}(0)=\max _{x_{N}=0} U_{x_{N}}\left(x^{\prime}, 0\right) \geq-\underline{u}^{\prime}(0)>0 .
$$


Moreover, it is easy to see that $U_{x_{N}}\left(x^{\prime}, 0\right)$ is negative if $\left|x^{\prime}\right|$ is large. Thus $U$ is positive near the origin and is negative if $\left|x^{\prime}\right|$ is large and $x_{N}>0$ is small. So we have proved that $U$ is a changing sign solution.

Next, we shall obtain a lower bound for $c_{\varepsilon}$.

Lemma 4.3. Suppose that $v_{\varepsilon}$ is a solution of (1.2) with $I_{\varepsilon}\left(v_{\varepsilon}\right)=c_{\varepsilon}$. Let $x_{\varepsilon}$ be the location of a maximum point of $u_{\varepsilon}$. Then

(i) we have $d\left(x_{\varepsilon}, \partial \Omega\right) \leq C \varepsilon$.

(ii) Suppose that $u_{\varepsilon}$ has another local maximum point $x_{\varepsilon}^{(1)}$. Then $\left|x_{\varepsilon}^{(1)}-x_{\varepsilon}\right| \leq C \varepsilon$.

(iii) For any $\theta>0$, there is a $\nu>0$, such that $v_{\varepsilon}(x) \leq C e^{-\nu\left|x-x_{\varepsilon}\right| / \varepsilon}, \forall x \in$ $\Omega \backslash B_{\theta}\left(x_{\varepsilon}\right)$.

Proof. Suppose that $\varepsilon^{-1} d\left(x_{\varepsilon}, \partial \Omega\right) \rightarrow+\infty$. Let $\bar{v}_{\varepsilon}(y)=v_{\varepsilon}\left(\varepsilon y+x_{\varepsilon}\right), y \in \Omega_{\varepsilon, x}=\{y:$ $\left.\varepsilon y+x_{\varepsilon} \in \Omega\right\}$. Then, from the upper bound for $c_{\varepsilon}$, we see that $\bar{v}_{\varepsilon}$ is bounded in $H^{1}\left(R^{N}\right)$. So we may assume (up to a subsequence) that

$$
\bar{v}_{\varepsilon} \rightarrow v_{0}, \text { in } C_{l o c}^{1}\left(R^{N}\right)
$$

and

$$
-\Delta v_{0}=f\left(v_{0}+a\right), \text { in } R^{N} .
$$

Thus, noting that $\frac{1}{2} g_{\varepsilon}(x, t) t-G_{\varepsilon}(x, t) \geq 0$, we see

$$
\begin{aligned}
I_{\varepsilon}\left(v_{\varepsilon}\right) & =\int_{\Omega}\left(\frac{1}{2} g_{\varepsilon}\left(x, v_{\varepsilon}\right) v_{\varepsilon}-G_{\varepsilon}\left(x, v_{\varepsilon}\right)\right) \\
& \geq \int_{B_{\varepsilon}\left(x_{\varepsilon}\right)}\left(\frac{1}{2} g_{\varepsilon}\left(x, v_{\varepsilon}\right) v_{\varepsilon}-G_{\varepsilon}\left(x, v_{\varepsilon}\right)\right) \\
& =\varepsilon^{N} \int_{B_{R}(0)}\left(\frac{1}{2} g_{\varepsilon}\left(\varepsilon y+x_{\varepsilon}, \bar{v}_{\varepsilon}\right) \bar{v}_{\varepsilon}-G_{\varepsilon}\left(\varepsilon y+x_{\varepsilon}, \bar{v}_{\varepsilon}\right)\right) \\
& =\varepsilon^{N}\left(\int_{B_{R}(0)}\left(\frac{1}{2} f\left(a+v_{0}\right) v_{0}-\left(F\left(a+v_{0}\right)-F(a)\right)\right)+o_{\varepsilon}(1)\right) \\
& \geq \varepsilon^{N}\left(A+o_{\varepsilon, R}(1)\right),
\end{aligned}
$$

where $o_{\varepsilon, R} \rightarrow 0$ as $\varepsilon \rightarrow 0$ and $R \rightarrow+\infty$. This is a contradiction to Proposition 4.1

Next, we prove (ii). Suppose that $\frac{\left|x_{\varepsilon}^{(1)}-x_{\varepsilon}\right|}{\varepsilon} \rightarrow+\infty$. Then it is easy to check that

$$
I_{\varepsilon}\left(v_{\varepsilon}\right) \geq \varepsilon^{N}(2 c+o(1)),
$$

where $o(1) \rightarrow 0$ as $\varepsilon \rightarrow 0$. This is a contradiction.

It remains to prove (iii).

First, we prove that as $\varepsilon \rightarrow 0, v_{\varepsilon} \rightarrow 0$ uniformly in $\Omega \backslash B_{\theta}\left(x_{\varepsilon}\right)$, for any $\theta>0$. In fact, suppose that there is $\bar{x}_{\varepsilon} \in \Omega \backslash B_{\theta}\left(x_{\varepsilon}\right)$, such that $v_{\varepsilon}\left(\bar{x}_{\varepsilon}\right) \geq c_{0}^{\prime}>0$. Then $v_{\varepsilon}\left(\varepsilon y+\bar{x}_{\varepsilon}\right) \rightarrow \bar{v}_{0} \neq 0$. As a result,

$$
\begin{aligned}
I_{\varepsilon}\left(v_{\varepsilon}\right) & =\int_{\Omega}\left(\frac{1}{2} g_{\varepsilon}\left(x, v_{\varepsilon}\right) v_{\varepsilon}-G_{\varepsilon}\left(x, v_{\varepsilon}\right)\right) \\
& \geq \int_{B_{\varepsilon}\left(x_{\varepsilon}\right)}\left(\frac{1}{2} g_{\varepsilon}\left(x, v_{\varepsilon}\right) v_{\varepsilon}-G_{\varepsilon}\left(x, v_{\varepsilon}\right)\right)+\int_{B_{\varepsilon R}\left(\bar{x}_{\varepsilon}\right)}\left(\frac{1}{2} g_{\varepsilon}\left(x, v_{\varepsilon}\right) v_{\varepsilon}-G_{\varepsilon}\left(x, v_{\varepsilon}\right)\right) \\
& \geq \varepsilon^{N}\left(2 c+o_{\varepsilon, R}(1)\right) .
\end{aligned}
$$

This is a contradiction. 
Next, from $-f^{\prime}\left(\underline{u}_{\varepsilon}\right) \geq 2 \nu_{0}>0$, for $x \in \Omega_{\varepsilon R}=:\{x \in \Omega, d(x, \partial \Omega) \geq \varepsilon R\}$ if $R>0$ is large enough, we can check easily by using the standard comparison theorem that

$$
v_{\varepsilon}(x) \leq C e^{-\sqrt{\nu_{0}}\left|x-x_{\varepsilon}\right| / \varepsilon}, \quad x \in \Omega_{\varepsilon R} .
$$

Suppose now that $x \in\left(\Omega \backslash \Omega_{\varepsilon R}\right) \backslash B_{\theta}\left(x_{\varepsilon}\right)$. Without loss of generality, we may assume $x_{\varepsilon}=0$. Denote $\bar{x} \in \partial \Omega$ such that $d(x, \partial \Omega)=|x-\bar{x}|$. Let $\xi(t)$ be the positive function defined in Appendix A. Let

$$
w_{\varepsilon}(x)=\xi\left(\frac{d(x, \partial \Omega)}{\varepsilon}\right) e^{-\bar{\nu}|\bar{x}| / \varepsilon}, \quad x \in \Omega \backslash \Omega_{\varepsilon R},
$$

where $\bar{\nu}>0$ is a small number. Noting that $\left\langle D e^{-\bar{\nu}|\bar{x}| / \varepsilon}, D d(x, \partial \Omega)=0\right\rangle$, we have for $x \in \Omega \backslash \Omega_{\varepsilon R}$,

$$
\begin{aligned}
& -\varepsilon^{2} \Delta w_{\varepsilon} \\
= & -\varepsilon^{2} e^{-\bar{\nu}|\bar{x}| / \varepsilon} \Delta \xi\left(\frac{d(x, \partial \Omega)}{\varepsilon}\right)-2 \varepsilon^{2}\left\langle D e^{-\bar{\nu}|\bar{x}| / \varepsilon}, D \xi\left(\frac{d(x, \partial \Omega)}{\varepsilon}\right)\right\rangle \\
& -\varepsilon^{2} \xi\left(\frac{d(x, \partial \Omega)}{\varepsilon}\right) \Delta e^{-\bar{\nu}|\bar{x}| / \varepsilon} \\
= & \left(-\xi^{\prime \prime}\left(\frac{d(x, \partial \Omega)}{\varepsilon}\right)+O(\varepsilon)\right) e^{-\bar{\nu}|\bar{x}| / \varepsilon}-\varepsilon^{2} \xi\left(\frac{d(x, \partial \Omega)}{\varepsilon}\right) \Delta e^{-\bar{\nu}|\bar{x}| / \varepsilon} \\
= & \left(-\xi^{\prime \prime}\left(\frac{d(x, \partial \Omega)}{\varepsilon}\right)+O(\varepsilon) \xi\left(\frac{d(x, \partial \Omega)}{\varepsilon}\right)\right) e^{-\bar{\nu}|\bar{x}| / \varepsilon}-\varepsilon^{2} \xi\left(\frac{d(x, \partial \Omega)}{\varepsilon}\right) \Delta e^{-\bar{\nu}|\bar{x}| / \varepsilon},
\end{aligned}
$$

since $\xi\left(\frac{d(x, \partial \Omega)}{\varepsilon}\right) \geq c_{0}^{\prime}>0$ for $x \in \Omega \backslash \Omega_{\varepsilon R}$. As a result,

$$
\begin{aligned}
& -\varepsilon^{2} \Delta w_{\varepsilon}-\left(f^{\prime}\left(\underline{u}_{\varepsilon}\right)-\tau\right) w_{\varepsilon} \\
= & -\varepsilon^{2} \xi\left(\frac{d(x, \partial \Omega)}{\varepsilon}\right) \Delta e^{-\bar{\nu}|\bar{x}| / \varepsilon} \\
& +\left(-\xi^{\prime \prime}\left(\frac{d(x, \partial \Omega)}{\varepsilon}\right)-\left(f^{\prime}\left(\underline{u}\left(\frac{d(x, \partial \Omega)}{\varepsilon}\right)\right)+O(\varepsilon)-\tau\right) \xi\left(\frac{d(x, \partial \Omega)}{\varepsilon}\right)\right) e^{-\bar{\nu}|\bar{x}| / \varepsilon} \\
= & \xi\left(\frac{d(x, \partial \Omega)}{\varepsilon}\right)\left(-\varepsilon^{2} \Delta e^{-\bar{\nu}|\bar{x}| / \varepsilon}+\left(3 \lambda_{0}+O(\varepsilon)-\tau\right) e^{-\bar{\nu}|\bar{x}| / \varepsilon}\right)>0, \quad \forall x \in \Omega \backslash \Omega_{\varepsilon R} .
\end{aligned}
$$

From $-\Delta v_{\varepsilon}-f^{\prime}\left(\underline{u}_{\varepsilon}\right) v_{\varepsilon}=O\left(\left|v_{\varepsilon}\right|^{2}\right)$ and $v_{\varepsilon} \rightarrow 0$ as $\varepsilon \rightarrow 0$ uniformly for $x \in \Omega \backslash B_{\theta / 2}(0)$, we see that $-\Delta v_{\varepsilon}-\left(f^{\prime}\left(\underline{u}_{\varepsilon}\right)-\tau\right) v_{\varepsilon} \leq 0$ if $x \in \Omega \backslash B_{\theta / 2}(0)$. So by comparison in the domain $\left(\Omega \backslash \Omega_{\varepsilon R}\right) \backslash B_{\theta / 2}(0)$, we have

$$
v_{\varepsilon}(x) \leq C \max _{z \in \Omega \cap \partial B_{\theta / 2}(0)}\left(w_{\varepsilon}(z)\right)^{-1} w_{\varepsilon}(x), \quad \forall x \in\left(\Omega \backslash \Omega_{\varepsilon R}\right) \backslash B_{\theta / 2}(0),
$$

since

$$
v_{\varepsilon} \leq C \max _{z \in \Omega \cap \partial B_{\theta / 2}(0)}\left(w_{\varepsilon}(z)\right)^{-1} w_{\varepsilon}(x) \quad \text { if } x \in \partial\left(\left(\Omega \backslash \Omega_{\varepsilon R}\right) \backslash B_{\theta / 2}(0)\right) .
$$

Thus, if $x \in\left(\Omega \backslash \Omega_{\varepsilon R}\right) \backslash B_{2 \theta}(0)$, then

$$
\begin{aligned}
v_{\varepsilon}(x) & \leq C \max _{z \in \Omega \cap \partial B_{\theta / 2}(0)}\left(w_{\varepsilon}(z)\right)^{-1} e^{-\bar{\nu} 3 \theta / 4 \varepsilon} e^{-\bar{\nu}|\bar{x}| / 4 \varepsilon} \\
& \leq C^{\prime} \max _{z \in \Omega \cap \partial B_{\theta / 2}(0)} e^{\nu|\bar{z}|} e^{-\bar{\nu} 3 \theta / 4 \varepsilon} e^{-\bar{\nu}|\bar{x}| / 4 \varepsilon} \leq C^{\prime} e^{-\bar{\nu}|\bar{x}| / 4 \varepsilon} .
\end{aligned}
$$

Proposition 4.4. Let $x_{\varepsilon}$ be a global maximum point of $v_{\varepsilon}$ and $\bar{x}_{\varepsilon} \in \partial \Omega$ be such that $\left|x_{\varepsilon}-\bar{x}_{\varepsilon}\right|=d\left(x_{\varepsilon}, \partial \Omega\right)$. Then,

$$
c_{\varepsilon} \geq \varepsilon^{N}\left(c-\varepsilon H\left(\bar{x}_{\varepsilon}\right) B(u)+o(\varepsilon)\right),
$$


where $u \in H_{0}^{1}\left(R_{+}^{N}\right)$ is a solution of $-\Delta u=f(\underline{u}+u)-f(\underline{u}), B(u)$ is defined as in Proposition 4.1.

Proof. Let $v_{\varepsilon}$ be a solution of (1.2) with $I_{\varepsilon}\left(v_{\varepsilon}\right)=c_{\varepsilon}$. Let $\bar{x}_{\varepsilon} \in \partial \Omega$ be such that $\left|\bar{x}_{\varepsilon}-x_{\varepsilon}\right|=d\left(x_{\varepsilon}, \partial \Omega\right)$. We assume that $\bar{x}_{\varepsilon}=0$.

Similar to Proposition 4.1 we define

$$
\tilde{v}_{\varepsilon}(x)=\eta(x) v_{\varepsilon}\left(x^{\prime}, x_{N}+\varphi\left(x^{\prime}\right)\right) \in H_{0}^{1}\left(R_{+}^{N}\right),
$$

where $\eta(x) \in C_{0}^{\infty}\left(B_{\delta}(0)\right)$, with $0 \leq \eta \leq 1, \eta=1$ for $x \in B_{\delta / 2}(0)$.

Denote $\bar{v}_{\varepsilon}=\tilde{v}_{\varepsilon}(\varepsilon y)$. Then

$$
\bar{v}_{\varepsilon} \rightarrow u, \quad \text { in } C_{l o c}^{1}\left(R_{+}^{N}\right),
$$

and

$$
\left\{\begin{array}{l}
-\Delta u=f\left(\underline{u}\left(x_{N}\right)+u\right)-f\left(\underline{u}\left(x_{N}\right)\right), \quad \text { in } R_{+}^{N}, \\
u \in H_{0}^{1}\left(R_{+}^{N}\right) .
\end{array}\right.
$$

Because $\bar{v}_{\varepsilon}$ attains its maximum at $y^{\prime}=0$, we see that $u(y)=u\left(\left|y^{\prime}\right|, y_{N}\right)$.

Since $v_{\varepsilon}$ is a mountain pass solution, we have

$$
\begin{aligned}
c_{\varepsilon} & =I_{\varepsilon}\left(v_{\varepsilon}\right)=\max _{t \geq 0} I_{\varepsilon}\left(t v_{\varepsilon}\right) \\
& =\max _{t \geq 0}\left[\frac{1}{2} t^{2} \varepsilon^{2} \int_{\Omega}\left|D v_{\varepsilon}\right|^{2}-\int_{\Omega}\left(F\left(\underline{u}_{\varepsilon}+t u_{\varepsilon}\right)-F\left(\underline{u}_{\varepsilon}\right)-f\left(\underline{u}_{\varepsilon}\right) t v_{\varepsilon}\right)\right] .
\end{aligned}
$$

Similar to the estimate of the upper bound for $c_{\varepsilon}$, using the fact that $v_{\varepsilon}$ is exponentially small outside $B_{\delta / 2}(0)$, we can prove that

$$
\begin{aligned}
& \frac{1}{2} \varepsilon^{2} \int_{\Omega}\left|D v_{\varepsilon}\right|^{2}=\frac{1}{2} \varepsilon^{2} \int_{\Omega \cap B_{\delta / 2}\left(x_{\varepsilon}\right)}\left|D v_{\varepsilon}\right|^{2}+O\left(e^{-\delta^{\prime} / \varepsilon}\right) \\
= & \varepsilon^{N}\left[\frac{1}{2} \int_{R_{+}^{N}}\left|D \bar{v}_{\varepsilon}\right|^{2}+\frac{\varepsilon H\left(\bar{x}_{\varepsilon}\right)}{4} \int_{x_{N}=0}\left|x^{\prime}\right|^{2}\left|D_{x_{N}} u\right|^{2}+o(\varepsilon)\right. \\
& \left.\quad+\frac{\varepsilon H\left(\bar{x}_{\varepsilon}\right)}{2} \int_{R_{+}^{N}}\left|x^{\prime}\right|^{2}\left(f\left(\underline{u}\left(x_{N}\right)+u\right)-f\left(\underline{u}\left(x_{N}\right)\right)-f^{\prime}\left(\underline{u}\left(x_{N}\right)\right) u\right) \underline{u}^{\prime}\left(x_{N}\right)\right] .
\end{aligned}
$$

Similarly, we have

$$
\begin{aligned}
\int_{\Omega} & \left(F\left(\underline{u}_{\varepsilon}+t v_{\varepsilon}\right)-F\left(\underline{u}_{\varepsilon}\right)-f\left(\underline{u}_{\varepsilon}\right) t v_{\varepsilon}\right) \\
= & \varepsilon^{N}\left[\int_{R_{+}^{N}}\left(F\left(\underline{u}\left(x_{N}\right)+t \bar{v}_{\varepsilon}\right)-F\left(\underline{u}\left(x_{N}\right)\right)-f\left(\underline{u}\left(x_{N}\right)\right) t \bar{v}_{\varepsilon}\right)\right. \\
& \left.+(N-1) \varepsilon \int_{R_{+}^{N}}\left(f\left(\underline{u}\left(x_{N}\right)+t u\right)-f\left(\underline{u}\left(x_{N}\right)\right)-f^{\prime}\left(\underline{u}\left(x_{N}\right)\right) t u\right) \psi\left(x_{N}\right) H\left(\bar{x}_{\varepsilon}\right)+o(\varepsilon)\right] .
\end{aligned}
$$


Thus

$$
\begin{aligned}
& I_{\varepsilon}\left(t v_{\varepsilon}\right) \\
&=\varepsilon^{N} {\left[\frac{t^{2}}{2} \int_{R_{+}^{N}}\left|D \bar{v}_{\varepsilon}\right|^{2}-\int_{R_{+}^{N}}\left(F\left(\underline{u}\left(x_{N}\right)+t \bar{v}_{\varepsilon}\right)-F\left(\underline{u}\left(x_{N}\right)\right)-f\left(\underline{u}\left(x_{N}\right)\right) t \bar{v}_{\varepsilon}\right)\right] } \\
&+ \varepsilon^{N+1} H\left(\bar{x}_{\varepsilon}\right)\left[\frac{1}{4} t^{2} \int_{x_{N}=0}\left|x^{\prime}\right|^{2}\left|D_{x_{N}} u\right|^{2}\right. \\
&+\frac{t^{2}}{2} \int_{R_{+}^{N}}\left|x^{\prime}\right|^{2}\left(f\left(\underline{u}\left(x_{N}\right)+u\right)-f\left(\underline{u}\left(x_{N}\right)\right)-f^{\prime}\left(\underline{u}\left(x_{N}\right)\right) u\right) \underline{u}_{\varepsilon}^{\prime}\left(x_{N}\right) \\
&\left.\quad-(N-1) \int_{R_{+}^{N}}\left(f\left(\underline{u}\left(x_{N}\right)+t u\right)-f\left(\underline{u}\left(x_{N}\right)\right)-f^{\prime}\left(\underline{u}\left(x_{N}\right)\right) t u\right) \psi\left(x_{N}\right)\right]+o\left(\varepsilon^{N+1}\right) \\
&= I_{\varepsilon 4}(t)+I_{\varepsilon 5}(t)+o\left(\varepsilon^{N+1}\right) .
\end{aligned}
$$

Choose $\tilde{t}_{\varepsilon}$ such that $I_{\varepsilon 4}\left(\tilde{t}_{\varepsilon}\right)=\max _{t \geq 0} I_{\varepsilon 4}(t)$. Since $\bar{v}_{\varepsilon} \in H_{0}^{1}\left(R_{+}^{N}\right)$, we see that

$$
I_{\varepsilon 4}\left(\tilde{t}_{\varepsilon}\right) \geq c .
$$

On the other hand, from $\bar{v}_{\varepsilon} \rightarrow u$ in $H_{0}^{1}\left(R_{+}^{N}\right)$, and since $u$ is a solution of $-\Delta u=$ $f(\underline{u}+u)-f(\underline{u})$, we can deduce easily that $\tilde{t}_{\varepsilon} \rightarrow 1$ as $\varepsilon \rightarrow 0$. Thus, $I_{\varepsilon 5}\left(\tilde{t}_{\varepsilon}\right)=$ $I_{\varepsilon 5}(1)+o\left(\varepsilon^{N+1}\right)$. As a result,

$$
c_{\varepsilon}=\max _{t \geq 0} I_{\varepsilon}\left(t v_{\varepsilon}\right) \geq I_{\varepsilon}\left(\tilde{t}_{\varepsilon} v_{\varepsilon}\right) \geq \varepsilon^{N}\left(c-\varepsilon H\left(\bar{x}_{\varepsilon}\right) B(u)+o(\varepsilon)\right),
$$

where $u \in H_{0}^{1}\left(R_{+}^{N}\right)$ is a solution of $-\Delta u=f(\underline{u}+u)-f(\underline{u})$. So we have completed the proof of this proposition.

Now we are ready to prove the following results.

Theorem 4.5. Let $v_{\varepsilon}$ be a positive mountain pass solution of (1.2). We have

(i) For any local maximum point $x_{\varepsilon}$ of $v_{\varepsilon}$, we have $d\left(x_{\varepsilon}, \partial \Omega\right) \leq C \varepsilon$. If $v_{\varepsilon}$ has another local maximum point $x_{\varepsilon}^{(1)}$, then $\left|x_{\varepsilon}-x_{\varepsilon}^{(1)}\right| \leq C \varepsilon$. Moreover, for any $\theta>0$, there is a $\nu>0$, such that $\left|v_{\varepsilon}(x)\right| \leq C e^{-\nu\left|x-x_{\varepsilon}\right| / \varepsilon}$ for $x \in \Omega \backslash B_{\theta}\left(x_{\varepsilon}\right)$.

(ii) For any sequence of $\varepsilon$, there is a subsequence $\varepsilon_{j} \rightarrow 0$, such that $x_{j} \rightarrow x_{0} \in \partial \Omega$ with $H\left(x_{0}\right)=\max _{x \in \partial \Omega} H(x)$, where $x_{j}$ is any local maximum point of $v_{\varepsilon}$, and $H(x)$ is the mean curvature of $\partial \Omega$ at $x$.

Proof. It is easy to see (i) follows from Lemma 4.3 To prove (ii), we can combine Propositions 4.1 and 4.4 to obtain

$$
-H\left(x_{0}\right) \leq-\max _{x \in \partial \Omega} H(x)+o(1) .
$$

As a result, $H\left(x_{0}\right)=\max _{x \in \partial \Omega} H(x)$.

Proof of Theorem 1.1, Let $z_{\varepsilon}$ be a global maximum point of $v_{\varepsilon}$. Then $u_{\varepsilon}\left(\varepsilon y+z_{\varepsilon}\right)$ converges in $C_{\text {loc }}^{1}\left(R_{+}^{N}\right)$ to $U(y)=\underline{u}\left(y_{N}\right)+u$, where $u$ is the mountain pass solution of (3.1). By Remark 4.2 we know that $\max _{y \in R_{+}^{N}} U(y)>0$. Thus (i) follows. It also follows from Remark 4.2 that $U_{x_{N}}(0)>0$. So we see that $\frac{\partial}{\partial \nu} u_{\varepsilon}\left(\varepsilon y+z_{\varepsilon}\right)>0$ at $\bar{z}_{\varepsilon}$, where $\nu$ is the inward unit normal of $\partial \Omega$ at $\bar{z}_{\varepsilon}, \bar{z}_{\varepsilon} \in \partial \Omega_{\varepsilon}$ satisfies $\left|z_{\varepsilon}-\bar{z}_{\varepsilon}\right|=$ $d\left(z_{\varepsilon}, \partial \Omega_{\varepsilon}\right), \Omega_{\varepsilon}=\left\{y: \varepsilon y+z_{\varepsilon} \in \Omega\right\}$. As a result, $u_{\varepsilon}\left(\varepsilon y+z_{\varepsilon}\right)>0$ in a neighbourhood of $\bar{z}_{\varepsilon}$ and (vi) follows. On the other hand, (ii) follows from (i) of Theorem 4.5

To prove (iii), we claim that for any local maximum point $x_{\varepsilon}$ of $u_{\varepsilon}$ with $u_{\varepsilon}\left(x_{\varepsilon}\right) \geq$ $\bar{c}_{0}>0$, we have $\left|x_{\varepsilon}-z_{\varepsilon}\right| \leq C \varepsilon$, where $z_{\varepsilon}$ is a local maximum point of $v_{\varepsilon}$. In fact, suppose that there is a sequence of $x_{\varepsilon}$, such that $\varepsilon^{-1}\left|x_{\varepsilon}-z_{\varepsilon}\right| \rightarrow+\infty$ as $\varepsilon \rightarrow 0$ for 
any local maximum point $z_{\varepsilon}$ of $v_{\varepsilon}$. Then $v_{\varepsilon} \geq \bar{c}_{0}$ in a small neighbourhood of $x_{\varepsilon}$. So we can blow up $v_{\varepsilon}$ at $x_{\varepsilon}$ and see that the energy of $v_{\varepsilon}$ is strictly larger than $c_{\varepsilon}$. So we get a contradiction. See the proof of Lemma 4.3. Thus (iii) follows from (ii) of Theorem 4.5 .

\section{EXISTENCE OF INTERIOR PEAK SOLUTIONS}

In this section, we shall briefly prove the existence of interior peak solutions for (1.2) and estimate the number of such solutions. For simplicity, we only discuss the case $f(t)=(t-a)^{p-1}-(t-a)$. So the mountain pass solution of (3.5) is nondegenerate. See [24].

As we see in Section 4, the main contribution to the energy of $I_{\varepsilon}\left(v_{\varepsilon}\right)$ comes from the error term in the expansion of $\underline{u}_{\varepsilon}$ near the boundary of $\Omega$. To construct the interior peak solution for (1.2), we need the following proposition.

Proposition 5.1. We have

$$
\underline{u}_{\varepsilon}(x)=a+e^{-\sqrt{-f^{\prime}(a)}(d(x, \partial \Omega)+o(1)) / \varepsilon},
$$

for any $x \in \Omega$ with $\varepsilon^{-1} d(x, \partial \Omega)$ large. Here, oo(1) $\rightarrow 0$ as $\varepsilon \rightarrow 0$.

Proof. See the proof of Theorem 2.1 in $[8]$.

Let $w$ be the unique positive solution of (3.5), which is nondegenerate. Define

$$
w_{\varepsilon, x}(y)=w\left(\frac{y-x}{\varepsilon}\right) .
$$

Let $P_{\varepsilon, \Omega} w_{\varepsilon, x} \in H_{0}^{1}(\Omega)$ be the solution of

$$
-\Delta v-f^{\prime}(a) v=f\left(a+w_{\varepsilon, x}\right)-f^{\prime}(a) w_{\varepsilon, x} .
$$

Using Proposition [5.1, we can prove

\section{Proposition 5.2.}

$$
I\left(P_{\varepsilon, \Omega} w_{\varepsilon, x}\right)=\varepsilon^{N} A-\tau_{\varepsilon, x}+O\left(e^{-(1+\sigma) \sqrt{-f^{\prime}(a)} d(x, \partial \Omega) / \varepsilon}\right),
$$

where $\tau_{\varepsilon, x}=\int_{\Omega}\left(f\left(a+w_{\varepsilon, x}\right)-f^{\prime}(a) w_{\varepsilon, x}\right)\left(\underline{u}_{\varepsilon}-a\right)$. Moreover, $\tau_{\varepsilon, x}$ satisfies

$$
c_{0} e^{-(1+\theta) \sqrt{-f^{\prime}(a)} d(x, \partial \Omega) / \varepsilon} \leq \tau_{\varepsilon, x} \leq c_{1} e^{-(1-\theta) \sqrt{-f^{\prime}(a)} d(x, \partial \Omega) / \varepsilon},
$$

for any $\theta>0$, and $c_{0}>0$ and $c_{1}>0$ are some constants.

Proof. The proof of this proposition is similar to that of Step 1 of Lemma 3.1 and thus we omit it.

By direct calculation, it is not difficult to prove the following proposition (see for example [10]):

\section{Proposition 5.3.}

$$
\begin{aligned}
& I\left(\sum_{j=1}^{k} P_{\varepsilon, \Omega} w_{\varepsilon, x_{j}}\right) \\
= & \sum_{j=1}^{k} I\left(P_{\varepsilon, \Omega} w_{\varepsilon, x_{j}}\right)-\int_{\Omega}\left(F_{a}\left(\sum_{j=1}^{k} w_{\varepsilon, x_{j}}\right)-\sum_{j=1}^{k} F_{a}\left(w_{\varepsilon, x_{j}}\right)-\sum_{i<j} f_{a}\left(w_{\varepsilon, x_{i}}\right) w_{\varepsilon, x_{j}}\right) \\
& +O\left(\sum_{j=1}^{k} e^{-(1+\sigma) \sqrt{-f^{\prime}(a)} d\left(x_{j}, \partial \Omega\right) / \varepsilon}+\sum_{i \neq j} e^{-(1+\sigma) \sqrt{-f^{\prime}(a)}\left|x_{j}-x_{i}\right| / \varepsilon}\right),
\end{aligned}
$$


where $f_{a}(t)=f(a+t), F_{a}(t)=\int_{0}^{t} f_{a}(\tau) d \tau$.

Propositions 5.2 and 5.3 show that the energy of the approximate multipeak solution $\sum_{j=1}^{k} P_{\varepsilon, \Omega} w_{\varepsilon, x_{j}}$ will become larger if the peak $x_{j}$ moves away from the boundary of $\Omega$, or if a pair of peak $\left(x_{i}, x_{j}\right)$ moves away from each other. Thus this estimate is similar to that for the interior peak solution of the Neumann problem [29]. But one should note that in Proposition 5.2 the first small term is $e^{-\sqrt{-f^{\prime}(a)} d(x, \partial \Omega) / \varepsilon}$, instead of $e^{-2 \sqrt{-f^{\prime}(a)} d(x, \partial \Omega) / \varepsilon}$ as in the Neumann problem.

Arguing in exactly the same way as in [7, 29], we have

Theorem 5.4. For any positive integer $k$, there is an $\varepsilon_{0}>0$, such that for any $\varepsilon \in\left(0, \varepsilon_{0}\right]$, (1.2) has a solution of the form

$$
v_{\varepsilon}=\sum_{j=1}^{k} P_{\varepsilon, \Omega} w_{\varepsilon, x_{\varepsilon, j}}+\varphi_{\varepsilon},
$$

where $\varphi_{\varepsilon}$ satisfies

$$
\begin{aligned}
& \int_{\Omega} \varepsilon^{2}\left|D \varphi_{\varepsilon}\right|^{2}+\varphi_{\varepsilon}^{2} \\
= & \varepsilon^{N} O\left(\sum_{j=1}^{k} e^{-(1+\sigma) \sqrt{-f^{\prime}(a)} d\left(x_{\varepsilon, j}, \partial \Omega\right) / \varepsilon}+\sum_{i \neq j} e^{-(1+\sigma) \sqrt{-f^{\prime}(a)}\left|x_{\varepsilon, j}-x_{\varepsilon, i}\right| / \varepsilon}\right) .
\end{aligned}
$$

Furthermore, we have

(i) if $k=1$, the number of the interior single peak solutions is at least Cat $(\Omega)$, and there is at least one single peak solution with its peak near the global maximum point of the distance function $d(x, \partial \Omega)$;

(ii) if $k \geq 2$, the number of the interior $k$ peak solutions is at least $N$, and there $i s$ at least one $k$ peak solution of the form (5.1) such that $\left(x_{\varepsilon, 1}, \cdots, x_{\varepsilon, k}\right) \rightarrow$ $\left(x_{0,1}, \cdots, x_{0, k}\right)$ and $\left(x_{0,1}, \cdots, x_{0, k}\right)$ is a maximum point of the function

$$
\min \left(d\left(x_{j}, \partial \Omega\right),\left|x_{i}-x_{j}\right|, i, j=1, \cdots, k, i \neq j\right) .
$$

Remark 5.5. The results here look similar to those in the Neumann problem. But for the interior multipeak solution of (1.2), we see that the locations of the peaks are different from those for the Neumann problem, where there is a positive interior peak solution, whose peaks are near a maximum point of the function

$$
\min \left(2 d\left(x_{j}, \partial \Omega\right),\left|x_{i}-x_{j}\right|, i, j=1, \cdots, k, i \neq j\right) \text {. }
$$

\section{Appendix A.}

Let $u$ be a positive solution of (3.1). In this section, we shall prove that $u$ decays exponentially as $|x| \rightarrow+\infty$. Since we do not assume that $f^{\prime}(t)$ is negative in $[a, 0]$, we cannot use the comparison theorem as usual to obtain the decay estimate. More work is needed.

Proposition A.1. Suppose that $u$ is a positive solution of (3.1). Then there are $C>0$ and $\lambda_{0}>0$, such that $u(x) \leq C e^{-\sqrt{\lambda_{0}}|x|}$.

Proof. Choose $R>0$ large enough, such that $f^{\prime}\left(\underline{u}\left(x_{N}\right)\right)<-2 \mu_{0}<0$ for $x_{N} \geq R$. Here $\mu_{0}>0$ is a small constant. By a standard comparison argument, we can get easily that

$$
u(x) \leq C e^{-\sqrt{\mu_{0}}|x|}, \quad \forall x_{N} \geq R .
$$


On the other hand, by Lemma 2.1, we have

$$
\int_{0}^{+\infty}\left(\left|\xi^{\prime}\right|^{2}-f^{\prime}(\underline{u}(t)) \xi^{2}\right) d t \geq 4 \lambda_{0} \int_{0}^{+\infty}\left(\left|\xi^{\prime}\right|^{2}+\xi^{2}\right) d t, \quad \forall \xi \in H_{0}^{1}((0,+\infty)),
$$

for some $\lambda_{0}>0$. So we see that the following problem has a positive solution $\xi(t)$ :

$$
\left\{\begin{array}{l}
-\xi^{\prime \prime}-f^{\prime}(\underline{u}(t)) \xi=3 \lambda_{0} \xi, \quad t \in(0, R) \\
\xi(0)=\xi(R)=1
\end{array}\right.
$$

In fact, let $\bar{\xi}(t)=\xi(t)-1$. By the Lax-Milgram theorem, we see that the following problem has a unique solution:

$$
\left\{\begin{array}{l}
-\bar{\xi}^{\prime \prime}-f^{\prime}(\underline{u}(t)) \bar{\xi}-3 \lambda_{0} \bar{\xi}=-f^{\prime}(\underline{u}(t))-3 \lambda_{0}, \quad t \in(0, R), \\
\xi(0)=\xi(R)=0 .
\end{array}\right.
$$

Thus (A.2) has a unique solution. Besides, using $\varphi=\xi^{-}=\min (0, \xi) \in H_{0}^{1}((0,1))$ as a test function, we see $\xi^{-}=0$. Hence, $\xi$ is nonnegative. Suppose that the minimum of $\xi$ equals 0 . Then $\xi^{\prime}=0$ at the minimum point. By the uniqueness of the ordinary differential equation, $\xi=0$. This is a contradiction. So $\xi$ is positive.

Let $v=C e^{-\sqrt{\lambda_{0}}\left|x^{\prime}\right|} \xi\left(x_{N}\right), x_{N} \in[0, R]$. Then

$$
\begin{aligned}
& -\Delta v-\left(f^{\prime}(\underline{u}(t))+\lambda_{0}\right) v \\
= & \xi\left(x_{N}\right)\left(-C \Delta e^{-\sqrt{\lambda_{0}}\left|x^{\prime}\right|}+C e^{-\sqrt{\lambda_{0}}\left|x^{\prime}\right|} \frac{-\xi^{\prime \prime}\left(x_{N}\right)-\left(f^{\prime}(\underline{u}(t))+\lambda_{0}\right) \xi\left(x_{N}\right)}{\xi\left(x_{N}\right)}\right) \\
= & \xi\left(x_{N}\right)\left(-C \Delta e^{-\sqrt{\lambda_{0}}\left|x^{\prime}\right|}+2 \lambda_{0} C e^{-\sqrt{\lambda_{0}}\left|x^{\prime}\right|}\right)>0,
\end{aligned}
$$

if $\left|x^{\prime}\right| \geq R_{1}>0$ is large enough.

Since $u \in H_{0}^{1}\left(R_{+}^{N}\right)$, we see that $u(x) \rightarrow 0$ as $|x| \rightarrow+\infty$. Thus we may choose $R_{1}>0$ large enough, such that $\frac{g_{1}(x, u(x))}{u(x)} \leq \lambda_{0}$ for $\left|x^{\prime}\right| \geq R_{1}$. As a result,

$$
-\Delta u-\left(f^{\prime}\left(\underline{u}\left(x_{N}\right)\right)+\lambda_{0}\right) u<0, \quad \text { if }\left|x^{\prime}\right| \geq R_{1} .
$$

Thus we see

$$
-\Delta(u-v)-\left(f^{\prime}(\underline{u}(t))+\lambda_{0}\right)(u-v)<0, \quad \text { if }\left|x^{\prime}\right| \geq R_{1} \text { and } x_{N} \in[0, R] .
$$

In view of (A.1), we see that we can choose $C>0$ large enough, such that $u(x) \leq$ $v(x)$ if $\left|x^{\prime}\right| \leq R_{1}$ and $x_{N} \in[0, R]$, or $x_{N}=0$, or $x_{N}=R$ (we can always choose $\left.\lambda_{0}<\mu_{0}\right)$. Let $\eta=(u-v)^{+}$if $x_{N} \in[0, R]$, and $\eta=0$ if $x_{N} \geq R$. Because $u \leq v$ for $x_{N}=R$, we see that $\eta \in H_{0}^{1}\left(R_{+}^{N}\right)$. Thus,

$$
\begin{aligned}
& \int_{R_{+}^{N}}\left(\left|D(u-v)^{+}\right|^{2}-\left(f^{\prime}(\underline{u}(t))+\lambda_{0}\right)\left|(u-v)^{+}\right|^{2}\right) \\
= & \int_{\left\{\left|x^{\prime}\right| \geq R_{1}\right\} \cap\left\{x_{N} \in[0, R]\right\}}\left(D(u-v)^{+} D \eta-\left(f^{\prime}(\underline{u}(t))+\lambda_{0}\right)(u-v)^{+} \eta\right) \leq 0,
\end{aligned}
$$

which implies $(u-v)^{+}=0$. That is, $u \leq v$. Here we have used the natural generalization of Lemma 2.1 to the half space.

Acknowledgment. The authors would like to thank Prof. Sweers for a useful conversation. 


\section{REFERENCES}

1. A.Ambrosetti and P.Rabinowitz Dual variational methods in critical point theory and applications, J. Funct. Anal., 14(1973), 349-381. MR 51:6412

2. H.Berestycki, L.Caffarelli and L.Nirenberg, Further qualitative properties for elliptic equations in unbounded domains, Ann. Scuola Norm. Sup. Pisa C1. Sci., 25, 69-94(1997). MR 2000e:35053

3. D.Cao, E.N.Dancer, E.Noussair and S.Yan, On the existence and profile of multi-peaked solutions to singularly perturbed semilinear Dirichlet problems, Discrete and Continuous Dynamical Systems, 2(1996), 221-236. MR 96m:35095

4. D.Cao and T.Küpper, On the existence of the multipeaked solutions to a semilinear Neumman problem, Duke Math. J. 97(1999), 261-300. MR 2000a:35064

5. P.Clément and G.Sweers, Existence and multiplicity results for a semilinear eigenvalue problem, Ann. Scuola Norm. Sup. Pisa, 14(1987), 97-121. MR 89j:35053

6. E.N.Dancer, On the number of positive solutions of weakly non-linear elliptic equations when a parameter is large, Proc. London Math. Soc., 53(1986), 429-452. MR 88c:35061

7. E.N. Dancer, K.Y.Lam and S. Yan, The effect of the graph topology on the existence of multipeak solutions for nonlinear Schrödinger equation, Abstract and Appl. Anal., 3(1998), 293-318. MR 2001g:35231

8. E.N.Dancer and J.Wei, On the profile of solutions with two sharp layers to a singularly perturbed semilinear Dirichlet problem, Proc. Royal Soc. Edinburgh, 127A(1997), 691-701. MR 98i:35012

9. E.N.Dancer and J.Wei, On the location of spikes of solutions with two sharp layers for a singularly perturbed semilinear Dirichlet problem, J. Diff. Equations, 157(1999), 82-101. MR 2000j:35017

10. E.N. Dancer and S. Yan, Multipeak solutions for a singularly perturbed Neumann problem, Pacific J. Math., 189(1999), 241-262. MR 2000d:35010

11. E.N.Dancer and S.Yan, A singularly perturbed elliptic problem in bounded domains with nontrivial topology, Adv. Diff. Equations, 4(1999), 347-368. MR 2000d:35009

12. E.N. Dancer and S. Yan, Interior and boundary peak solutions for a mixed boundary value problem, Indiana University Math. J., 48(1999), 1177-1212. MR 2001f:35146

13. M. Del Pino and P.Felmer, Spike-layered solutions of singularly perturbed elliptic problems in a degenerate setting, Indiana Univ. Math.J., 48(1999), 883-898. MR 2001b:35027

14. B. Gidas, W.M. Ni and L. Nirenberg, Symmetry and related properties via the maximum principle, Comm. Math. Phys. 68(1979), 209-243. MR 80h:35043

15. D.Gilbarg and N.S.Trudinger, Elliptic partial differential equations of second order, second edition, Springer-Verlag, Berlin, Heidelberg, New York, Tokyo, 1983. MR 86c:35035

16. C. Gui and J.Wei, Multiple interior peak solutions for some singularly perturbed Neumann problem, J. Diff. Equations 158(1999), 1-27. MR 2000g:35035

17. C. Gui, J.Wei and M.Winter, Multiple boundary peak solutions for some singularly perturbed Neumann problem, Ann. Inst. H. Poincaré Anal., Non Linéaire, 17(2000), 47-82. MR 2001a:35018

18. E.F.Keller and L.A.Segel, Initiation of slime mold aggregation viewed as an instability, J. Theoret. Bio., 26(1970), 399-415.

19. J.Jang, On the spike solutions of singularly perturbed semilinear Dirichlet problem, J.Diff. Equations, 114(1994), 370-395. MR 95i:35099

20. P.L.Lions, The concentration compactness principle in the calculus of variations, the locally compact case, I,II, Ann. Inst. H. Poincaré, Anal. Non Linéaire, 1(1984), 109-145, 223-283. MR 87e:49035a MR 87e:49035b

21. Y.Y. Li, On a singularly perturbed equation with Neumann boundary condition, Comm. PDE, 23(1998), 487-545. MR 2000a:35013

22. H.Meinhardt, Models of biological pattern formation, Academic Press, 1982.

23. W.M. Ni and I. Takagi, On the shape of the least energy solution to a semilinear Neumann problem, Comm. Pure Appl. Math. 41(1991), 819-851. MR 92i:35052

24. W.M. Ni and I. Takagi, Locating the peaks of least energy solutions to a semilinear Neumann problem, Duke Math. J.70(1993), 247-281. MR 94h:35072

25. W.M. Ni, I. Takagi and J.Wei, On the locations and profile of spike-layer solutions to a singularly perturbed semilinear Dirichlet problem, intermediate solution, Duke Math. J., 94(1998), 597-618. MR 99h:35011 
26. W.M.Ni and J.Wei, On the location and profile of spike-layer solutions to singularly perturbed semilinear Dirichlet problems, Comm. Pure Appl. Math. 48(1995), 731-768. MR 96g:35077

27. G.Sweers, Some results for a semilinear elliptic problem with a large parameter, Proceedings of the First International Conference on Industrial and Applied Mathematics, Paris-La Villette, 1987, pp. 109-116. MR 89d:35065

28. J.Wei, On the construction of single-peaked solutions to a singularly perturbed semilinear Dirichlet problem, J. Diff. Equations, 129(1996), 315-333. MR 97f:35015

29. S. Yan, On the number of the interior multipeak solutions for singularly perturbed Neumann problems, Topological Methods in Nonlinear Anal., 12(1999), 61-78. MR 2001c:35024]

School of Mathematics and Statistics, University of Sydney, New South Wales 2006, Australia

E-mail address: normd@maths.usyd.edu.au

School of Mathematics and Statistics, University of Sydney, New South Wales 2006, Australia

E-mail address: shusen@maths.usyd.edu.au 